\title{
A generating process of geomagnetic drifting field
}

\author{
Takesi Yukutake and Hisayoshi Shimizu* (D)
}

\begin{abstract}
The geomagnetic field is comprised of drifting and standing fields. The drifting field has two remarkable features. One is predominance of sectorial harmonics when the field is expressed in a spherical harmonic series, and the other is uniform drift rate irrespective of harmonics. We consider that the drifting field is a product of interaction of the core flow with the axial dipole field near the surface of the core. The key to the predominance of sectorial harmonics is in the boundary condition on the electric current at the core-mantle boundary. If we take the mantle to be an electrical insulator, the electric current normal to the boundary must vanish. This strongly constrains the surface flow. The toroidal flow becomes the flow with the sectorial harmonics predominant. Then, the sectorial toroidal flow, interacting with the axial dipole field, induces the poloidal field in which the sectorial harmonics are predominant. This is the observed type of drifting field. The uniform drift rate, the second nature of the drifting field, seems to suggest that the surface part of the core is rotating westwards as a whole. Subsequently, the sectorial type toroidal flow embedded in the westward-rotating surface layer is considered as the cause of the drifting field.
\end{abstract}

Keywords: Geomagnetic secular variation, Westward drift, Sectorial harmonics, Flow in Earth's core

\section{Introduction}

\section{Geomagnetic secular variation and westward drift}

It is well known that some specific features of the geomagnetic field are drifting westwards, while many other features are standing at the same place, changing their intensity. Take, for example, the intense focus of the vertical component anomaly in the equatorial region near the western coast of African continent observed at the Earth's surface. It has been moving westwards steadily for the past several hundred years (e.g., Bullard et al. 1950; Yukutake and Tachinaka 1968a). At the core surface, a focus corresponding to the African focus was confirmed to exist near the equator and drifting westwards (Bloxham et al. 1989; Jackson 2003; Finlay and Jackson 2003). Besides, the drifting foci are distributed in a narrow equatorial region (Jackson 2003; Finlay and Jackson 2003). These observations seem to suggest that the westward drift in the low latitudes is a special feature characteristic in the equatorial region.

*Correspondence: shimizu@eri.u-tokyo.ac.jp

Earthquake Research Institute, University of Tokyo, Yayoi 1-1-1, Bunkyo-ku, Tokyo 113-0032, Japan
From this, a question arises whether the drift might be a local phenomenon limited in the equatorial region. In the atmospheric and oceanic sciences, the equatorially trapped wave is a well-known phenomenon (e.g., Bretherton 1964; Matsuno 1964; Longuet-Higgins 1968; Pedlosky 1987; Zhang 1993). However, it is not certain whether such confinement occurs when a magnetic field exists. Employing an equatorial $\beta$-plane approximation for the surface layer of the core, Bergman (1993) examined how the equator-trapped wave was modified in the presence of the dipole field. Although full induction equations were not incorporated in his formulation, he found that the magnetic field released the wave from confinement in the equatorial region, and that the wave extended its undulation to higher latitudes.

Investigations of the core flow that produces the observed magnetic field and its secular variation are now being conducted intensively. These are mainly based on the frozen flux approximation (Roberts and Scott 1965) that ignores magnetic diffusion and regards the observed secular variation is due to the advection of field at the surface of the core. The frozen flux approximation is widely applied to the several decade data that 
include the satellite observation. Further assumptions are made such as tangentially geostrophy or quasi-geostrophy (see Bloxham and Jackson 1991; Holme 2015; Finlay et al. 2010, for review). In the tangentially geostrophic assumption, core surface flow has been estimated by assuming that the Lorentz force is negligibly weak at the core surface and the geostrophic balance holds. In the quasi-geostrophic assumption, the flow is pursued that produces the observed type of magnetic field and its temporal variation at the core surface by assuming that the flow in the core consists of quasi-geostrophic columnar flow (e.g., Pais and Jault 2008; Gillet et al. 2009; Amit and Pais 2013). On the other hand, the flow and field of numerical geodynamo models were examined whether they could generate the field compatible with the observation at the surface of the core. The model by Aubert et al. (2013), for example, which is based on the gravitational coupling between the inner core and the mantle, is a successful one. Latitudinal and longitudinal distributions of the secular variation similar to the observations are obtained. Furthermore, endeavors are being made to assimilate the observed field to the flow model in the core to pursue the development of the magnetic field (Kuang et al. 2009, 2010; Tangborn and Kuang 2015; Barrois et al. 2017, 2018). One of the issues common to these studies is azimuthal flow near the core surface, corresponding to the drifting of the African focus observed at the Earth's surface. Strong westward flows are commonly obtained in the equatorial region of Atlantic hemisphere (e.g., Gillet et al. 2015; Finlay et al. 2016), although the existence of eastward drifting phase is also suggested, for example, in the higher latitudes (Finlay and Jackson 2003). However, detailed structure such as the equator- symmetric nature of the drifting field, which is one of the characteristic features of the drifting field (Yukutake and Shimizu 2015), is not well recovered yet.

Although the westward drift appears to be restricted to special features when the main field is examined, distribution of the temporal variation of the field, secular variation, is different. The westward drift is also recognized in the pattern of the secular variation as well. However, drifting features cover wider region, extending from equatorial region to higher latitudes (Yukutake and Tachinaka 1968b; Yukutake and Shinizu 2015). The secular variation seems to suggest that the westward drift is more like a global phenomenon.

Employing the Gauss coefficients of the geomagnetic potential expressed in spherical harmonic series, Yukutake and Tachinaka (1969) decomposed the geomagnetic field into drifting and standing fields. The drifting field is the field drifting westwards for the past several hundred years, while the standing field is the field staying at the same location. In the drifting field, two features are remarkable (Yukutake 1981; Yukutake and Shimizu 2015). One is the predominance of sectorial harmonics where the degree $(n)$ and order $(m)$ are equal. The vertical component (or magnetic potential) is symmetric about the equator with its maximum at the equator. The other remarkable feature is about the drift velocity. It is nearly uniform, about $0.3^{\circ}$ year, irrespective of harmonics. On the other hand, the standing field is largely anti-symmetric about the equator and its intensity is stronger than the drifting field. Accordingly, when the drifting and standing fields are synthesized, in the total field the drifting field is mostly concealed by the standing field and observable only in a narrow equatorial region as the African magnetic anomaly (Yukutake and Shimizu 2016).

In this study, we attempt to clarify the nature of the drifting field based on the drifting and standing field model. Particularly, we concentrate on the characteristic feature, predominance of sectorial harmonics in the drifting field.

\section{Outline of the generating process}

We consider that the westward drift is a manifestation of hydromagnetic process near the surface of the core, where the dipole field is dominant. From a nature of the drifting field, we assume that the surface layer of the core is rotating westwards as a whole. The uniform drift rate, one of the characteristic features of the drifting field, implies that the drift velocity is nondispersive. It has long been a matter of controversy whether the westward drift is due to a material flow or a hydromagnetic wave (Bullard et al. 1950; Hide 1966; Holme 2015). The uniform drift rate seems to favor the material flow, in which the field is generated and conveyed together. Because the hydromagnetic wave is a dispersive wave in general, the wave velocity is highly dependent on harmonics. We assume here that the surface layer is rotating westwards, in which the sectorial type field is produced by interaction of fluid flow with the dipole field.

If we take the mantle to be electrically insulating, electrical currents normal to the core-mantle boundary must vanish at the boundary. This leads to predominance of sectorial harmonics in the toroidal flow. This type of toroidal flow, interacting with the dipole field, induces the sectorial type of poloidal field (Yokoyama and Yukutake 1989; Yukutake and Yokoyama 1988). This is the type of the observed drifting field for the lower harmonics up to $n=m=6$ for the past 400 years (e.g., Yukutake and Shimizu 2015). When this process occurs in the layer rotating westwards, the induced sectorial poloidal field is observed as the drifting field that is characterized by the sectorial harmonics. In the poloidal flow system, on the other hand, the fluid motion is confined on a meridional plane in the surface layer aligned in the north-south 
direction. The meridional flow induces the zonal poloidal field that is immune to rotation. Then, it is impossible to observe the field produced by the poloidal flow as the drifting field. Consequently, only the sectorial field generated by the toroidal flow is observed as the drifting field. This process will be confirmed with a simplistic model in the following sections.

\section{Theory and methods}

\section{Model and equations}

We consider a three-layer model shown in Fig. 1. Region I is the mantle, and Regions II and III form the core. The drifting field is generated in the surface layer of the core (Region II). No fluid flow is assumed in the innermost part (Region III). The core is rotating westward relative to the mantle. The mantle is taken to be electrically insulating.

The electrical conductivity $\left(\sigma_{i}\right)$ is assigned to each region as follows.

$$
\begin{array}{lll}
\text { Region I (mantle) } & 1<r^{\prime} & \sigma_{1}=0, \\
\text { Region II (core) } & r_{1}^{\prime} \leq r^{\prime} \leq 1 & \sigma_{2}=\infty \text { or } \sigma, \\
\text { Region III (core) } & r^{\prime}<r_{1}^{\prime} \quad \sigma_{3}=\sigma,
\end{array}
$$

where $r^{\prime}=r / r_{0}$ and $r_{0}$ is the radius of the core.

Assume that the whole Earth rotates at an angular velocity $\Omega$ with an axial dipole field $\mathbf{B}$. They are written with spherical coordinates $(r, \theta, \varphi)$ as

$$
\begin{aligned}
& \boldsymbol{\Omega}=(\cos \theta,-\sin \theta, 0) \Omega_{0} \\
& \mathbf{B}=(2 \cos \theta, \sin \theta, 0) B_{0} / r^{\prime 3}
\end{aligned}
$$

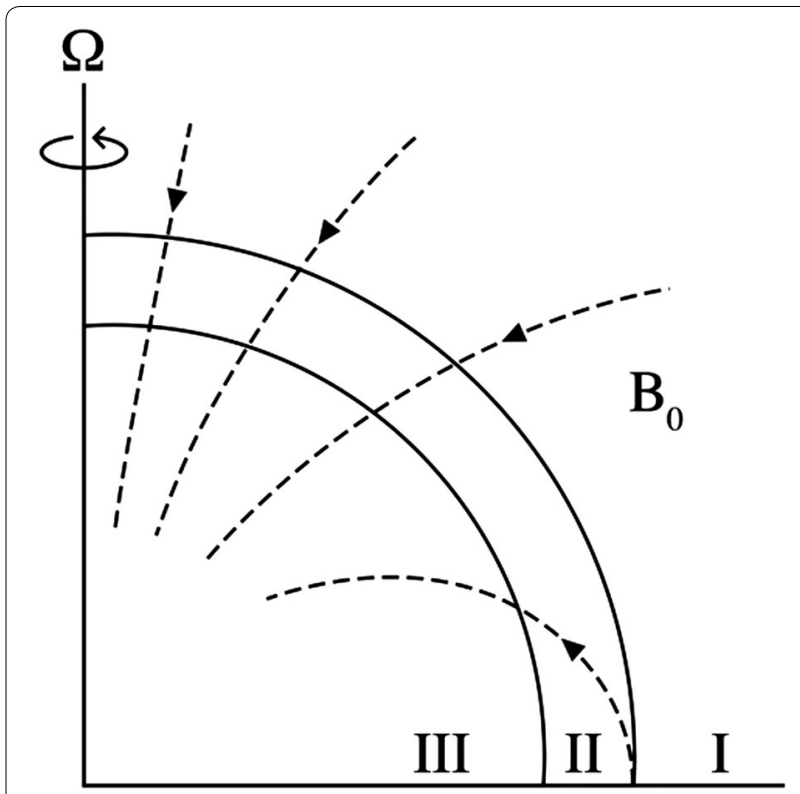

Fig. 1 Model of the core and mantle
Region II and III rotate at an angular velocity of $\omega_{0}$ relative to the mantle (Region I).

Followings are the equations to be solved for respective regions. Since Region I is electrically insulating, the induced magnetic field $\mathbf{b}$ in the mantle becomes a potential field. Therefore,

$$
\begin{aligned}
& \nabla^{2} \mathbf{b}=0, \\
& \mathbf{b}=-\operatorname{grad} V, \\
& \operatorname{div} \mathbf{b}=0,
\end{aligned}
$$

In Region II, we assume that the magnetic field is generated by action of electrically conducting fluid flow. Then,

$$
\begin{aligned}
\frac{\partial \mathbf{v}}{\partial t}+2 \boldsymbol{\Omega} \times \mathbf{v}= & -\frac{1}{\rho} \operatorname{grad} p+\frac{1}{\rho \mu} \operatorname{curl} \mathbf{b} \times \mathbf{B} \\
& +\frac{1}{\rho \mu} \operatorname{curl} \mathbf{B} \times \mathbf{b}-\frac{\rho^{\prime}}{\rho} \mathrm{g} \hat{\mathbf{r}}+v \nabla^{2} \mathbf{v},
\end{aligned}
$$

$$
\frac{\partial \mathbf{b}}{\partial t}=\operatorname{curl}(\mathbf{v} \times \mathbf{B})+\eta \nabla^{2} \mathbf{b},
$$

$$
\operatorname{divv}=0, \quad \operatorname{divb}=0,
$$

$$
\frac{\partial \rho^{\prime}}{\partial t}=-\beta v_{r}+\kappa \nabla^{2} \rho^{\prime}
$$

$$
\left.\begin{array}{l}
\beta=\frac{\mathrm{d} \rho_{s}}{\mathrm{~d} r} \\
\rho=\rho_{s}+\rho
\end{array}\right\}
$$

$$
\begin{aligned}
& \rho_{s}(r)=\rho_{0}-\frac{\beta}{2}\left(\frac{r}{r_{0}}\right)^{2}, \\
& \mathrm{~g}(r)=\mathrm{g}_{0}\left(\frac{r}{r_{0}}\right),
\end{aligned}
$$

where $\mathbf{v}$ is velocity, $\rho$ is density, $v$ is kinematic viscosity, $\kappa$ is thermal diffusivity, $\eta$ is magnetic diffusivity that is $\eta=1 /(\sigma \mu), \mu$ is magnetic permeability, and $\mathrm{g}$ is gravitational acceleration. In Region III, we assume that the fluid is stationary. Then, $\mathbf{b}$ must satisfy

$$
\frac{\partial \mathbf{b}}{\partial t}=\eta \nabla^{2} \mathbf{b}
$$

$$
\operatorname{divb}=0 .
$$


In Regions II and III, we employ toroidal and poloidal vector expression for velocity (v) and magnetic field (b) as

$$
\begin{aligned}
& \mathbf{v}=\mathbf{v}_{T}+\mathbf{v}_{S}, \\
& \mathbf{v}_{T}=\operatorname{curl}(\psi \mathbf{r}), \quad \mathbf{v}_{S}=\operatorname{curl}[\operatorname{curl}(\Phi \mathbf{r})], \\
& \mathbf{b}=\mathbf{b}_{T}+\mathbf{b}_{S}, \\
& \mathbf{b}_{T}=\operatorname{curl}(T \mathbf{r}), \quad \mathbf{b}_{S}=\operatorname{curl}[\operatorname{curl}(S \mathbf{r})],
\end{aligned}
$$

where suffix $\mathrm{T}$ indicates toroidal vector, and $\mathrm{S}$ poloidal vector. Also, $\psi$ and $\Phi$ are scalar functions for the velocity potential, while $T$ and $S$ are scalar functions for the magnetic field. With these scalar functions, we rewrite the equations.

In Region II, taking curl and curl.curl of (6), (7), (9), we obtain their radial components as follows;

$$
\begin{gathered}
{\left[\frac{\partial}{\partial t}-v \nabla^{2}\right] \frac{L^{2} \psi}{r}-2 \Omega_{0} \frac{1}{r} \frac{\partial \psi}{\partial \varphi}-\frac{2 \Omega_{0}}{r^{2}}\left[D_{A} \hat{\Phi}^{\prime}-D_{B} L^{2} \Phi\right]} \\
=\frac{B_{0}}{\rho \mu} \frac{r_{0}^{3}}{r^{5}}\left[2 D_{A} \hat{T}^{\prime}+D_{B} L^{2} T\right]+\frac{2 B_{0}}{\rho \mu} \frac{r_{0}^{3}}{r^{4}} \frac{\partial}{\partial \varphi} \nabla^{2} S
\end{gathered}
$$

$$
\begin{gathered}
{\left[\frac{\partial}{\partial t}-v \nabla^{2}\right] \frac{L^{2} \nabla^{2} \Phi}{r}-2 \Omega_{0} \frac{1}{r} \frac{\partial \nabla^{2} \Phi}{\partial \varphi}+\frac{2 \Omega_{0}}{r^{2}}\left[D_{A} \hat{\psi}^{\prime}-D_{B} L^{2} \psi\right]} \\
=\frac{B_{0}}{\rho \mu} \frac{r_{0}^{3}}{r^{6}} \frac{\partial}{\partial \varphi}\left[L^{2} \hat{T}^{\prime}+r^{4}\left\{2 \frac{\partial}{\partial r}\left(\frac{\hat{T}^{\prime}}{r^{3}}\right)-\frac{\partial}{\partial r}\left(\frac{L^{2} T}{r^{3}}\right)\right\}\right] \\
\quad-\frac{B_{0}}{\rho \mu} \frac{r_{0}^{3}}{r^{2}}\left[2 D_{A}\left(\frac{\partial}{\partial r} r \frac{\nabla^{2} S}{r^{3}}\right)+D_{B}\left(\frac{\nabla^{2} S}{r^{3}}\right)\right]+\frac{g_{0}}{\rho_{s} r_{0} r} L^{2} \rho^{\prime}
\end{gathered}
$$

$$
\begin{aligned}
& {\left[\frac{\partial}{\partial t}-\eta \nabla^{2}\right] \frac{L^{2} T}{r}=\frac{B_{0} r_{0}^{3}}{r^{2}}\left[2 D_{A} \frac{\partial}{\partial r}\left(r \frac{\psi}{r^{3}}\right)+D_{B} L^{2}\left(\frac{\psi}{r^{3}}\right)\right]} \\
& -\frac{2 B_{0} r_{0}^{3}}{r^{2}} \frac{\partial}{\partial \varphi}\left[\nabla^{2} \Phi-\frac{3}{r}\left\{\frac{\partial \Phi}{\partial r}-\left[L^{2}-1\right] \frac{\Phi}{r}\right\}\right] \\
& {\left[\frac{\partial}{\partial t}-\eta \nabla^{2}\right] \frac{L^{2} S}{r}=\frac{2 B_{0} r_{0}^{3}}{r^{4}} \frac{\partial \psi}{\partial \varphi}} \\
& +\frac{B_{0} r_{0}^{3}}{r^{5}}\left[2 D_{A} \frac{\partial}{\partial r}(r \Phi)+D_{B} L^{2} \Phi\right],
\end{aligned}
$$

$$
\left[\frac{\partial}{\partial t}-\kappa \nabla^{2}\right] \rho^{\prime}=-\beta \frac{L^{2} \Phi}{r},
$$

where

$$
\mathrm{L}^{2} F=-\left[\frac{1}{\sin \theta} \frac{\partial}{\partial \theta}\left(\sin \theta \frac{\partial}{\partial \theta}\right)+\frac{1}{\sin ^{2} \theta} \frac{\partial^{2}}{\partial \varphi^{2}}\right] F,
$$

$$
\begin{aligned}
& \nabla^{2} F=\frac{1}{r} \frac{\partial^{2}}{\partial r^{2}}(r F)-\frac{L^{2} F}{r^{2}}, \\
& \hat{F}^{\prime}=\frac{\partial}{\partial r}(r F), \\
& \mathrm{D}_{\mathrm{A}} F=\left[\cos \theta \mathrm{L}^{2}+\sin \theta \frac{\partial}{\partial \theta}\right] F, \\
& \mathrm{D}_{\mathrm{B}} F=\left[2 \cos \theta \mathrm{L}^{2}+\sin \theta \frac{\partial}{\partial \theta}\right] F
\end{aligned}
$$

Similarly, Eq. (13) in Region III is written as,

$$
\left[\frac{\partial}{\partial t}-\eta \nabla^{2}\right] \frac{\mathrm{L}^{2} T}{r}, \quad\left[\frac{\partial}{\partial t}-\eta \nabla^{2}\right] \frac{\mathrm{L}^{2} S}{r} .
$$

In the followings, we use $r^{\prime}$ for $r$ and $\eta^{\prime}$ for $\eta$,

$$
r^{\prime}=\frac{r}{r_{0}}, \quad \eta^{\prime}=\frac{\eta}{r_{0}^{2}} \text {. }
$$

We assume that Regions II and III are rotating relative to Region I. However, for the moment, we adopt the coordinate system that is fixed to the rotating Regions II and III.

For the velocity potential, we take the following form with spherical polar coordinates,

$$
\begin{aligned}
& \psi=\sum_{n} \sum_{m} a_{n}^{m} G_{n}\left(\alpha_{n} r^{\prime}\right) P_{n}^{m}(\cos \theta) e^{i m \varphi} e^{i \omega t} \\
& G_{n}\left(\alpha_{n} r^{\prime}\right)=A_{n} j_{n}\left(\alpha_{n} r^{\prime}\right)+B_{n} n_{n}\left(\alpha_{n} r^{\prime}\right) \\
& \Phi=\sum_{n} \sum_{m} b_{n}^{m} E_{n}\left(\alpha_{n} r^{\prime}\right) P_{n}^{m}(\cos \theta) e^{i m \varphi} e^{i \omega t} \\
& E_{n}\left(\alpha_{n} r^{\prime}\right)=C_{n} r^{\prime} j_{n}\left(\alpha_{n} r^{\prime}\right)+D_{n} r^{\prime} n_{n}\left(\alpha_{n} r^{\prime}\right)
\end{aligned}
$$

where $j_{n}\left(\alpha_{n} r^{\prime}\right)$ and $n_{n}\left(\alpha_{n} r^{\prime}\right)$ are spherical Bessel functions.

In this study, we take $v=0, \kappa=0$. Regarding the electrical conductivity, we study two cases. One is the case where the electrical conductivity in Region II is infinite, and the other is the case where it is finite.

\section{Boundary conditions}

Since $v=0$, the surface of the top layer of the core and the boundary between Regions II and III are stress-free boundaries. Then, we have

$$
\frac{\partial}{\partial r^{\prime}}\left(\frac{\psi}{r^{\prime}}\right)=0 \quad \text { at } r^{\prime}=1 \text { and } r^{\prime}=r_{1}^{\prime},
$$




$$
\Phi=0, \frac{\partial^{2} \Phi}{\partial r^{\prime 2}}=0 \quad \text { at } r^{\prime}=1 \text { and } r^{\prime}=r_{1}^{\prime} .
$$

In order to satisfy the condition (23), $\alpha_{n}$ in $\psi$ must be the roots of

$$
\eta_{n}\left(\alpha_{n}\right) \zeta_{n}\left(\alpha_{n} r_{1}^{\prime}\right)-\zeta_{n}\left(\alpha_{n}\right) \eta_{n}\left(\alpha_{n} r_{1}^{\prime}\right)=0
$$

where

$\left.\begin{array}{l}\eta_{n}\left(\alpha_{n} r^{\prime}\right)=\frac{1}{2 n+1} \frac{\alpha_{n}}{r^{\prime}}\left\{(n-1) j_{n-1}\left(\alpha_{n} r^{\prime}\right)-(n+2) j_{n+1}\left(\alpha_{n} r^{\prime}\right)\right\} \\ \zeta_{n}\left(\alpha_{n} r_{1}^{\prime}\right)=\frac{1}{2 n+1} \frac{\alpha_{n}}{r^{\prime}}\left\{(n-1) n_{n-1}\left(\alpha_{n} r^{\prime}\right)-(n+2) n_{n+1}\left(\alpha_{n} r^{\prime}\right)\right\}\end{array}\right\}$

Note that there is infinite number of roots for $\alpha_{n}\left(\alpha_{n, i}, i=1,2,3 \ldots\right)$.

In order to satisfy (24), $\beta_{n}$ must be the roots of the following equation,

$$
j_{n}\left(\beta_{n}\right) n_{n}\left(\beta_{n} r_{1}^{\prime}\right)-n_{n}\left(\beta_{n}\right) j_{n}\left(\beta_{n} r_{1}^{\prime}\right)=0 .
$$

Then, we have

$$
E_{n}\left(\beta_{n} r^{\prime}\right)=C_{n} r^{\prime} j_{n}\left(\beta_{n}\right)\left[\frac{j_{n}\left(\beta_{n} r^{\prime}\right)}{j_{n}\left(\beta_{n}\right)}-\frac{n_{n}\left(\beta_{n} r^{\prime}\right)}{n_{n}\left(\beta_{n}\right)}\right] .
$$

The magnetic field produced in Region II diffuses out into Region I (mantle) and Region III. Magnetic flux density should be continuous at $r^{\prime}=1$ and $r^{\prime}=r_{1}^{\prime}$. The electric current normal to the boundary should be zero at $r^{\prime}=1$, and continuous at $r^{\prime}=r_{1}^{\prime}$.

Since the normal component of the electric current is given by

$$
j_{r}=\frac{L^{2} T^{\prime}}{r^{\prime}}
$$

the boundary condition on the electric current at the core-mantle boundary is

$$
\frac{L^{2} T^{\prime}}{r^{\prime}}=0 \quad \text { at } r^{\prime}=1 .
$$

\section{Results}

\section{Infinite conductivity in Region II}

\section{Predominance of sectorial harmonics in the toroidal flow}

The flow structure in the surface layer is greatly affected by the boundary condition on the electric current at the core-mantle boundary. Stress-free flow is further restricted by the boundary condition on the electric currents. If we take the case where the electric current induced by the toroidal flow and the current by the poloidal flow respectively satisfy the boundary condition, sectorial harmonics dominate in the velocity potential of toroidal flow, and the velocity potential of poloidal flow is restricted to zonal harmonics.

When the electrical conductivity of the generating layer is infinite, the boundary condition on the electric current (25) due to the poloidal potential $\Phi$ becomes

$$
\begin{aligned}
& \frac{2}{i \omega} \frac{B_{0}}{r^{\prime 4} r_{0}^{2}} \frac{\partial}{\partial \varphi}\left[\nabla^{\prime 2} \Phi-\frac{3}{r^{\prime}}\left\{\frac{\partial \Phi}{\partial r^{\prime}}-\left[L^{2}-1\right] \frac{\Phi}{r^{\prime}}\right\}\right]=0 \\
& \quad \text { at } r^{\prime}=1
\end{aligned}
$$

from the induction Eq. (17).

Under the free surface condition (24), this leads to

$$
\frac{\partial \Phi}{\partial \varphi}=0 \quad \text { at } r^{\prime}=1
$$

Namely, we obtain

$$
m=0 .
$$

Then, $\Phi$ is expressed only by zonal harmonics,

$$
\Phi=\sum_{n} b_{n}^{0} E_{n}\left(\beta_{n} r^{\prime}\right) P_{n}^{0}(\cos \theta) e^{i \omega t} .
$$

On the other hand, the condition on the current by toroidal flow $\psi$ leads to

$$
\frac{1}{i \omega} \frac{B_{0}}{r^{\prime 2} r_{0}}\left[2 D_{A} \frac{\partial}{\partial r^{\prime}}\left(r^{\prime} \frac{\psi}{r^{\prime 3}}\right)+D_{B} L^{2}\left(\frac{\psi}{r^{\prime 3}}\right)\right]=0 \quad \text { at } r^{\prime}=1 .
$$

By using the free surface condition on $\psi$, this becomes

$$
\begin{aligned}
& \frac{1}{\omega} \frac{1}{2 n+1} a_{n}^{m} G_{n}\left(\alpha_{n}\right)\left[(n-1)(n+1)(n+2)(n+m) P_{n-1}^{m}(\theta)\right. \\
& \left.\quad-(n-1) n(n+2)(n-m+1) P_{n+1}^{m}(\theta)\right]=0,
\end{aligned}
$$

and we can rewrite this as

$$
\frac{1}{\omega}\left[a_{n+1}^{m} u_{n}^{m} G_{n+1}\left(\alpha_{n+1}\right)-a_{n-1}^{m} v_{n}^{m} G_{n-1}\left(\alpha_{n-1}\right)\right] P_{n}^{m}(\theta)=0,
$$

where

$$
\begin{aligned}
& u_{n}^{m}=\frac{n(n+2)(n+3)(n+m+1)}{2 n+3}, \\
& v_{n}^{m}=\frac{(n-2)(n-1)(n+1)(n-m)}{2 n-1} .
\end{aligned}
$$

After some manipulation, we obtain

$$
a_{m+2 j+1}^{m}=0 \quad(j=0,1,2,3, \ldots)
$$

when $(n-m)$ is odd. This implies that the velocity potential function $\psi$ does not include the terms that are antisymmetric about the equator. 
On the other hand, when $(n-m)$ is even, we have

$$
\frac{a_{m+2 j_{\max }}^{m}}{a_{m}^{m}}=\prod_{j=1}^{j_{\max }} r_{m+2 j-1}^{m} \frac{G_{m}\left(\alpha_{m}\right)}{G_{m+2 j_{\max }}\left(\alpha_{m+2 j_{\max }}\right)} \quad(j=1,2,3, \ldots)
$$

where

$$
r_{m+2 j-1}^{m}=\frac{v_{m+2 j-1}^{m}}{u_{m+2 j-1}^{m}}
$$

This indicates that all $a_{m+2 j}^{m}$ are determined uniquely by $a_{m}^{m}$. Accordingly, except for the case of $a_{m}^{m}=0$, taking (30) into consideration, we obtain

$$
\psi=\sum_{m} \sum_{j} a_{m+2 j}^{m} G_{m+2 j}\left(\alpha_{m+2 j} r^{\prime}\right) P_{m+2 j}^{m}(\cos \theta) e^{i m \varphi} e^{i \omega t}
$$

for $j=0,1,2,3, \ldots$. This makes it clear that $\psi$ is a function symmetric about the equator.

Table 1 shows $a_{m+2 j}^{m}$ calculated using Eq. (31) in case where $r^{\prime}=0.9$ and $a_{m}^{m}=1$. It is remarkable that $a_{m}^{m}$ is predominant among $a_{m+2 j}^{m}$. This means function $\psi$ is well approximated by the sectorial terms.

\section{Magnetic field in the generating layer}

Let's consider the magnetic potential induced by the fluid motion that is constrained by the boundary condition on the electric current. Let $S_{\psi}$ be the poloidal magnetic potential produced by toroidal flow $\psi$, and $S_{\Phi}$ by poloidal flow $\Phi$. Then solving induction equation, we obtain

$$
\begin{aligned}
S_{\psi}= & 2 B_{0} \sum_{m} \sum_{j} a_{m+2 j}^{m} \frac{1}{\omega} \frac{m}{(m+2 j)(m+2 j+1)} \\
& \times \frac{1}{r^{\prime 3}} G_{m+2 j}\left(\alpha_{m+2 j} r^{\prime}\right) P_{m+2 j}^{m}(\cos \theta) e^{i m \varphi} e^{i \omega t} .
\end{aligned}
$$

With $a_{m+2 j}^{m}$ in Table 1 , we find that the sectorial term dominates in $S_{\psi}$ (see the equation with numerical coefficients given in "Appendix: Explicit expression of $S_{\psi}$ ").

Table 1 Relative amplitude of $a_{n}^{m}$ for $r_{1}^{\prime}=0.9$ and $i=1$

\begin{tabular}{lllll}
\hline & $\boldsymbol{a}_{\boldsymbol{m}}^{\boldsymbol{m}}$ & $\boldsymbol{a}_{\boldsymbol{m}+\mathbf{2}}^{\boldsymbol{m}}$ & $\boldsymbol{a}_{\boldsymbol{m}+\mathbf{4}}^{\boldsymbol{m}}$ & $\boldsymbol{a}_{\boldsymbol{m}+\mathbf{6}}^{\boldsymbol{m}}$ \\
\hline$m=2$ & 1 & $3.3 \times 10^{-2}$ & $1.5 \times 10^{-3}$ & $6.3 \times 10^{-5}$ \\
$m=3$ & 1 & $4.4 \times 10^{-2}$ & $1.9 \times 10^{-3}$ & $7.6 \times 10^{-5}$ \\
$m=4$ & 1 & $4.5 \times 10^{-2}$ & $1.9 \times 10^{-3}$ & $7.1 \times 10^{-5}$ \\
$m=5$ & 1 & $4.4 \times 10^{-2}$ & $1.8 \times 10^{-3}$ & $6.1 \times 10^{-5}$ \\
$m=6$ & 1 & $4.2 \times 10^{-2}$ & $1.6 \times 10^{-3}$ & $5.2 \times 10^{-5}$ \\
\hline
\end{tabular}

Infinite conductivity model
This is due to predominance of the sectorial term $a_{m}^{m}$ in $\psi$. The obtained $S_{\psi}$ is exactly the observed type of field as the drifting field. Therefore, it can be said that the predominance of the sectorial term in the drifting field is a product of the boundary condition on the electric current at the core-mantle boundary.

For the potential $S_{\Phi}$, poloidal flow $\left(\Phi_{n}^{m}\right)$ induces two types of poloidal magnetic field $S_{\Phi, n-1}^{m}, S_{\Phi, n+1}^{m}$. Since only the terms with $m=0$ are nonzero by the boundary condition for $\Phi, S_{\Phi}$ is also represented only by zonal harmonics as

$$
\begin{aligned}
S_{\Phi} & i B_{0} \sum_{n} b_{n}^{0} \frac{1}{\omega} \frac{1}{r^{\prime 4} r_{0}} \frac{1}{2 n+1}\left[( n + 1 ) \left\{(n-2) E_{n}\left(\beta_{n} r^{\prime}\right)\right.\right. \\
& \left.-2 \beta_{n} r^{\prime} E_{n}^{\prime}\left(\beta_{n} r^{\prime}\right)\right\} P_{n-1}^{0}(\cos \theta)-n\left\{(n+3) E_{n}\left(\beta_{n} r^{\prime}\right)\right. \\
& \left.\left.+2 \beta_{n} r^{\prime} E_{n}^{\prime}\left(\beta_{n} r^{\prime}\right)\right\} P_{n+1}^{0}(\cos \theta)\right] e^{i \omega t} .
\end{aligned}
$$

This result suggests that the field produced by the poloidal flow cannot be observed as the drifting field because the field is zonal. Therefore, only the sectorial field generated by the sectorial toroidal flow is observed as the drifting field.

Let $T_{\psi}$ be the toroidal magnetic field induced by toroidal flow $\psi$, and $T_{\Phi}$ be by poloidal flow $\Phi$. For the toroidal flow $\psi$ constrained by the boundary condition, $T_{\psi}$ becomes

$$
\begin{aligned}
T_{\psi}= & i B_{0} \sum_{m} \sum_{j} a_{m+2 j}^{m} \frac{1}{\omega} \frac{1}{r^{\prime 4} r_{0}} \frac{1}{2(m+2 j)+1} \cdot e^{i m \varphi} e^{i \omega t} . \\
& {\left[\frac { 2 ( m + 2 j + 1 ) ( m + j ) } { m + 2 j } \left\{(m+2 j+4) G_{m+2 j}\left(\alpha_{m+2 j} r^{\prime}\right)\right.\right.} \\
& \left.-2 \alpha_{m+2 j} r^{\prime} G_{m+2 j}^{\prime}\left(\alpha_{m+2 j} r^{\prime}\right)\right\} P_{m+2 j-1}^{m}(\cos \theta) \\
& -\frac{(m+2 j)(2 j+1)}{m+2 j+1}\left\{(m+2 j-3) G_{m+2 j}\left(\alpha_{m+2 j} r^{\prime}\right)\right. \\
& \left.\left.+2 \alpha_{m+2 j} r^{\prime} G_{m+2 j}^{\prime}\left(\alpha_{m+2 j} r^{\prime}\right)\right\} P_{m+2 j+1}^{m}(\cos \theta)\right] .
\end{aligned}
$$

Since the sectorial terms predominate, $T_{\psi}$ can be approximated by

$$
\begin{aligned}
T_{\psi}= & i B_{0} \sum_{j} a_{m}^{m} \frac{1}{\omega} \frac{1}{r^{\prime 4} r_{0}} \frac{1}{2 m+1} \cdot e^{i m \varphi} e^{i \omega t} . \\
& {\left[2(m+1)\{(m+4\}) G_{m}\left(\alpha_{m} r^{\prime}\right)\right.} \\
& \left.-2 \alpha_{m} r^{\prime} G_{m}^{\prime}\left(\alpha_{m} r^{\prime}\right)\right\} P_{m-1}^{m}(\cos \theta) \\
& -\frac{m}{m+1}\left\{(m-3) G_{m}\left(\alpha_{m} r^{\prime}\right)\right. \\
& \left.\left.+2 \alpha_{m} r^{\prime} G_{m}^{\prime}\left(\alpha_{m} r^{\prime}\right)\right\} P_{m+1}^{m}(\cos \theta)\right] .
\end{aligned}
$$


On the other hand, $T_{\Phi}$ becomes zero. It is because, as discussed in a previous section, $\frac{\partial \Phi}{\partial \varphi}=0$ due to the boundary

condition on the electric current. The induction equation becomes

$$
\frac{\partial}{\partial t} \frac{L^{2} T_{\Phi}}{r^{\prime}}=0
$$

Accordingly, we obtain

$$
T_{\Phi}=0 \text {. }
$$

This is notable. Toroidal magnetic field is induced solely by the toroidal flow $\psi$, and the poloidal flow $\Phi$ has no effect on generating the toroidal magnetic field.

\section{Characteristic frequency}

In order to obtain the intensity of magnetic field, it is necessary to estimate the frequency of the system, as is clear in (33) and (34). The model in this study is an oscillating system caused by imbalance between Coriolis and Lorentz forces. In the following, we estimate the characteristic frequency of the system.

Eliminating magnetic field in momentum equations, we obtain characteristic equations for the frequency. For the toroidal flow, they produce two separate classes of frequency. One is the inertial frequency $\left(\omega_{i}\right)$, and the other is the magnetostrophic frequency $\left(\omega_{m}\right)$. If we take $\Omega_{0}$ to be the rotational frequency of the Earth, and $\Omega_{B}$ as

$$
\Omega_{B}^{2}=\frac{B_{0}^{2}}{\rho \mu} \frac{1}{r_{0}^{2}},
$$

we have $\Omega_{B}^{2} \ll \Omega_{0}^{2}$ in our model. An order-of-magnitude estimate by a dimensional analysis gives

$$
\left.\begin{array}{l}
\omega_{i} \approx \Omega_{0}=O\left(10^{-4}\right) \quad 1 / \mathrm{s} \\
\omega_{m} \approx \frac{\Omega_{B}^{2}}{\Omega_{0}}=O\left(10^{-14}\right) \quad 1 / \mathrm{s}
\end{array}\right\}
$$

For the poloidal flow, the inertia-gravitational frequency $\left(\omega_{\mathrm{g}}\right)$ that is a kind of inertial frequency modified by gravitational and Lorentz forces is obtained as

$$
\omega_{g} \approx-\Omega_{0} \pm \sqrt{\Omega_{0}^{2}+\Omega_{g}^{2}}\left[1+\frac{1}{2} \frac{\Omega_{B}^{2}}{\Omega_{0}^{2}+\Omega_{B}^{2}}\right]=O\left(10^{-4}\right) \quad 1 / \mathrm{s}
$$

in case $\Omega_{g}^{2} \approx \Omega_{0}^{2}$, where

$$
\Omega_{g}^{2}=-\frac{\beta}{\rho} g
$$

Since the drifting field is the field induced by the toroidal flow, we here concentrate on the magnetostrophic mode, whose frequency is as low as $10^{-14} 1 / \mathrm{s}$.

Eliminating $T$ and $S$ in momentum Eqs. (15) and (16) by means of induction Eqs. (17) and (18), we multiply them by $G_{n}\left(\alpha_{n, j} r\right) P_{n^{\prime}}^{m^{\prime}}(\cos \theta) e^{i m^{\prime} \varphi} r^{2} \sin \theta \mathrm{d} r \mathrm{~d} \theta \mathrm{d} \varphi$, and integrate over interior of the spherical shell. Then, we obtain

$$
\left.\begin{array}{l}
\sum_{n} \sum_{m}\left[a_{n}^{m}\left\{\tilde{K}_{n}^{m}+\tilde{p}_{o, n}^{m}\right\}+a_{n+2}^{m} \tilde{p}_{a, n+2}^{m}+a_{n-2}^{m} \tilde{p}_{b, n-2}^{m}\right]=0 \\
\sum_{n} \sum_{m}\left[a_{n+1}^{m} \tilde{p}_{c, n+1}^{m}+a_{n-1}^{m} \tilde{p}_{d, n-1}^{m}\right]=0
\end{array}\right\}
$$

where

$$
\tilde{K}_{n}^{m}=\left\{\omega n(n+1)-2 m \Omega_{0}\right\} g g_{n}
$$

For the expressions of coefficients $\left\{\tilde{p}_{o, n}^{m}, \tilde{p}_{a, n+2}^{m}, \tilde{p}_{b, n-2}^{m}, \ldots\right\}$ see "Appendix: Coefficients in Eqs. (37) and (40)."

Since this is intractable, we here adopt a very crude approximation. We solve the following equation,

$$
\tilde{K}_{m}^{m}+\tilde{p}_{o, m}^{m}=0,
$$

and regard the obtained frequency as the representative frequency of this system. Explicit expression of (39) becomes

$$
\begin{aligned}
& \left\{\omega m(m+1)-2 m \Omega_{0}\right\} g g_{m}+\frac{\Omega_{B}^{2}}{\omega}\left\{\left(c_{t n 1}+\frac{4 m}{m+1}\right) g_{n}\right. \\
& \left.+\left(c_{t n 2}+\frac{16 m}{m+1}\right) d g_{m}+\left(c_{t n 3}-\frac{4 m}{m+1}\right) d_{2} g_{n}\right\}=0 .
\end{aligned}
$$

(For $\left\{c_{t n 1}, c_{t n 2}, c_{t n 3}, \mathrm{gg}_{m}, \mathrm{~g}_{n}, d \mathrm{~g}_{m}, d_{2} \mathrm{~g}_{n}\right\}$, see "Appendix: Coefficients in Eqs. (37) and (40).")

In $\left\{c_{t n 1}, c_{t n 2}, c_{t n 3}, \ldots\right\}$ of the above equation, $n$ should be taken to be $m(n=m)$. The obtained frequency is separated into two classes, a relatively high frequency of the order of $O\left(10^{-4}\right) 1 / \mathrm{s}$ and a low frequency lower than $O\left(10^{-10}\right) 1 / \mathrm{s}$. The higher frequency is inertial frequency, and the low frequency is magnetostrophic frequency. Approximate solution and numerical values when $m=2$ are shown as the following.

Inertial frequency

$$
\omega_{m}=\frac{2}{m+1} \Omega_{0},
$$

$$
\omega_{2}=\frac{2}{3} \Omega_{0}=0.486 \times 10^{-4} 1 / \mathrm{s} .
$$


Table 2 Characteristic magnetostrophic frequency $\omega_{m}$ for $r_{1}^{\prime}=0.9$ and $i=1$

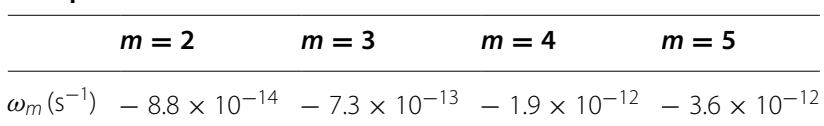

Infinite conductivity model

Magnetostropic frequency

$$
\begin{aligned}
\omega_{m}= & \frac{1}{2 m} \frac{\Omega_{B}^{2}}{\Omega_{0}}\left\{\left(c_{t n 1}+\frac{4 m}{m+1}\right) \frac{g_{m}}{g g_{m}}\right. \\
& \left.+\left(c_{t n 2}+\frac{16 m}{m+1}\right) \frac{d g_{m}}{g g_{m}}+\left(c_{t n 3}-\frac{4 m}{m+1}\right) \frac{d_{2} g_{m}}{g g_{m}}\right\}, \\
& \omega_{2}=-8.79 \times 10^{-14} 1 / \mathrm{s} .
\end{aligned}
$$

This is a very low frequency as initially guessed.

Numerical solutions for magnetostrophic frequency are listed in Table 2 for $r_{1}^{\prime}=0.9$ and $i=1$.

\section{Magnetic field in the mantle}

The magnetic field generated in Region II diffuses out into the mantle. The diffused field is derived by continuation of the normal component of magnetic flux density at the boundary.

Until here, the magnetic potentials $S_{\psi}$ and $S_{\Phi}$ have been obtained based on the coordinate system fixed in Region II that is rotating westwards. When the westward velocity $v$ is constant, $S_{\psi}$ in (33) and $S_{\Phi}$ in (34) are expressed in the coordinate system fixed to the mantle Region I as follows.

$$
\begin{aligned}
S_{\psi}= & 2 B_{0} \sum_{m} \sum_{j} a_{m+2 j}^{m} \frac{1}{\omega} \frac{m}{(m+2 j)(m+2 j+1)} \\
& \times \frac{1}{r^{\prime 3}} G_{m+2 j}\left(\alpha_{m+2 j} r^{\prime}\right) P_{m+2 j}^{m}(\cos \theta) e^{i m(v t+\varphi)} e^{i \omega t}, \\
S_{\Phi}= & i B_{0} \sum_{n} b_{n}^{0} \frac{1}{\omega} \frac{1}{r^{\prime 4} r_{0}} \frac{1}{2 n+1}\left[( n + 1 ) \left\{(n-2) E_{n}\left(\beta_{n} r^{\prime}\right)\right.\right. \\
& \left.-2 \beta_{n} r^{\prime} E_{n}^{\prime}\left(\beta_{n} r^{\prime}\right)\right\} P_{n-1}^{0}(\cos \theta)-n\left\{(n+3) E_{n}\left(\beta_{n} r^{\prime}\right)\right. \\
& \left.\left.+2 \beta_{n} r^{\prime} E_{n}^{\prime}\left(\beta_{n} r^{\prime}\right)\right\} P_{n+1}^{0}(\cos \theta)\right] e^{i \omega t} .
\end{aligned}
$$

Since the sectorial terms predominate, (41) is approximately

$$
S_{\psi}=2 B_{0} \sum_{m} a_{m}^{m} \frac{1}{\omega} \frac{1}{(m+1)} \frac{1}{r^{\prime 3}} G_{m}\left(\alpha_{m} r^{\prime}\right) P_{m}^{m}(\cos \theta) e^{i m(v t+\varphi)} e^{i \omega t}
$$

It is noted that (42) is the same as (34), which means that the zonal field is not affected by transformation of the coordinate system from rotating to static system.
In the mantle (Region I), the magnetic field consists of the fields originated from $S_{\psi}$ and $S_{\Phi}$. Let $V_{\psi}$ and $V_{\Phi}$ be

$$
\left.\begin{array}{rl}
V_{\psi} & =\sum_{n} \sum_{m} V_{\psi, n}^{m} P_{n}^{m}(\cos \theta) e^{i m \varphi} e^{i \omega^{\prime} t} \\
V_{\Phi} & =\sum_{n} \sum_{m}^{m} V_{\Phi, n}^{m} P_{n}^{m}(\cos \theta) e^{i m \varphi} e^{i \omega^{\prime} t}
\end{array}\right\}
$$

where $V_{\psi}$ is the potential of $S_{\psi}$ origin, and $V_{\Phi}$ of $S_{\Phi}$ origin. Then, the boundary condition at $r^{\prime}=1$ leads to

$$
\begin{aligned}
V_{\psi}= & 2 B_{0} \sum_{m} \sum_{j} a_{m+2 j}^{m} \frac{1}{\omega} \frac{m}{m+2 j+1} G_{m+2 j}\left(\alpha_{m+2 j}\right) \\
& \times\left(\frac{1}{r^{\prime}}\right)^{m+2 j+1} P_{m+2 j}^{m}(\cos \theta) e^{i m(v t+\varphi)} e^{i \omega t}
\end{aligned}
$$

$$
\begin{aligned}
V_{\Phi}= & i B_{0} \sum_{n} b_{n}^{0} \frac{1}{\omega} \frac{1}{r_{0}} \frac{n+1}{2 n+1}\left[( n - 1 ) \left\{(n-2) E_{n}\left(\beta_{n}\right)\right.\right. \\
& \left.-2 \beta_{n} E_{n}^{\prime}\left(\beta_{n}\right)\right\}\left(\frac{1}{r^{\prime}}\right)^{n} P_{n-1}^{0}(\cos \theta)-n\left\{(n+3) E_{n}\left(\beta_{n}\right)\right. \\
& \left.\left.+2 \beta_{n} E_{n}^{\prime}\left(\beta_{n}\right)\right\}\left(\frac{1}{r^{\prime}}\right)^{n+2} P_{n+1}^{0}(\cos \theta)\right] e^{i \omega t}
\end{aligned}
$$

Here, we note that the rigidly rotating core satisfies the boundary conditions both on the free surface flow and on the electric current at the core-mantle boundary despite the surface velocity being only in the westward direction.

\section{Toroidal velocity}

We make an order-of-magnitude estimate of the toroidal velocity. By making use of (45), we can estimate the velocity that induces the observed drifting field to find that very slow velocity is capable of producing the observed field. Figure 2 shows the observed drifting field

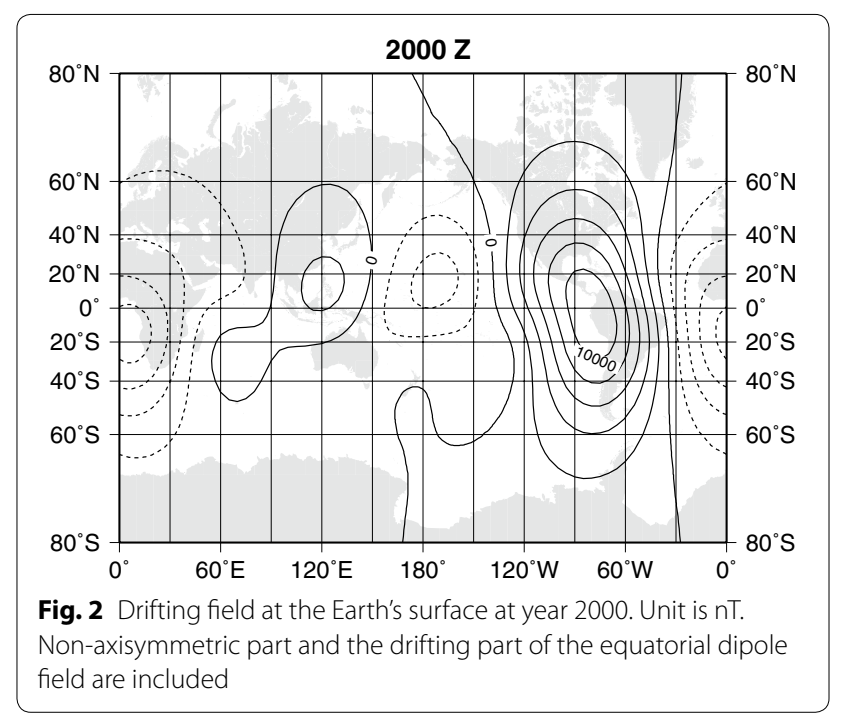




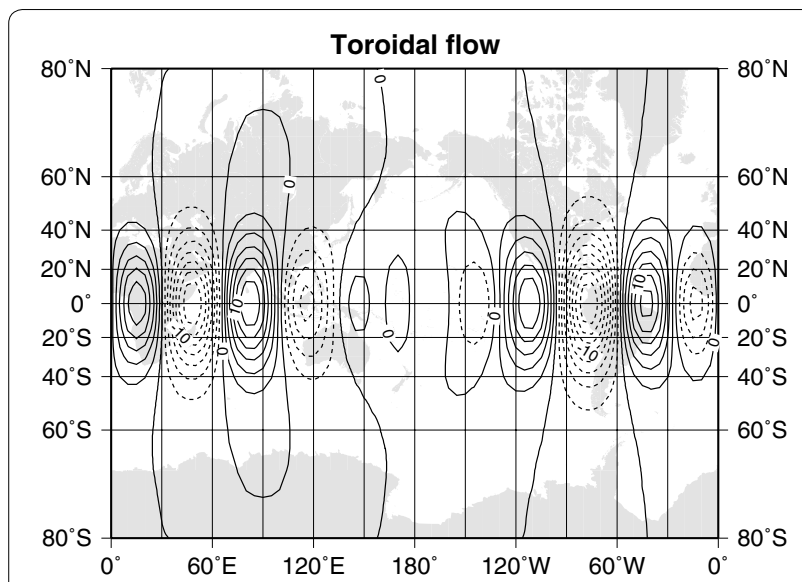

Fig. 3 Velocity potential $\psi$ at the core surface estimated from the drifting field at year $2000 \mathrm{AD}$. Unit is $10^{-6} \mathrm{~cm} / \mathrm{s}$. Infinite conductivity is assumed in Region II

for 2000AD. The toroidal velocity that produces sectorial parts of the drifting field in Fig. 2 is calculated and depicted in Fig. 3. Solid line indicates anti-clockwise motion, while dotted line clockwise. It is noticeable that small-scale flows as expressed by sectorial harmonics $n=m=6$ are predominant. In Fig. 4, the maximum northward velocity for each harmonic order at the equator is plotted. The maximum velocity increases with the harmonic order. For $m=2$, for example, the velocity is $2.1 \times 10^{-6} \mathrm{~cm} / \mathrm{s}$, while for $m=6$, it is $4.7 \times 10^{-5} \mathrm{~cm} / \mathrm{s}$. This increasing trend means that, in order to generate the observable field, the higher velocities are necessary for the smaller scale fields. It should be noted, however, that the drifting-standing field in the present

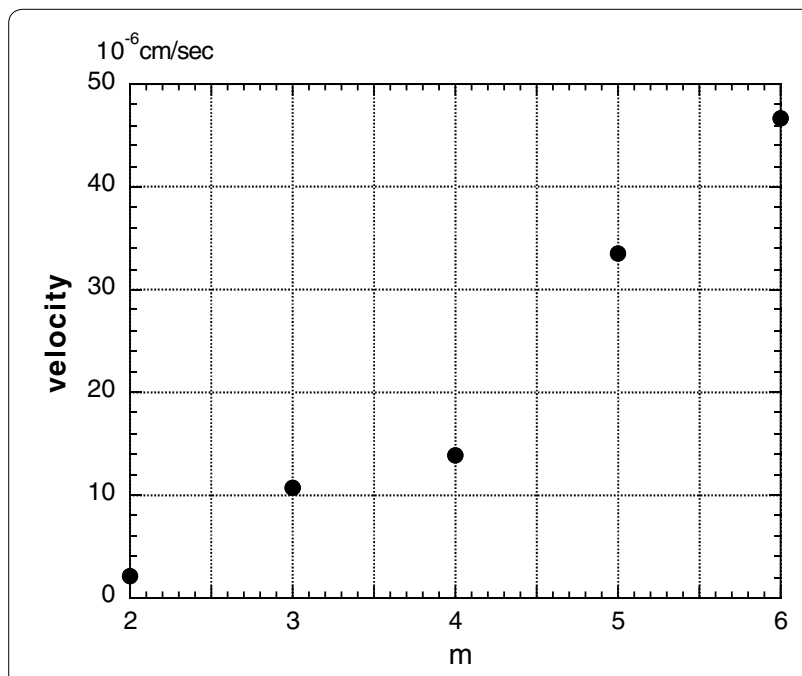

Fig. 4 Maximum velocity at the equator on the core surface. In the unit of $10^{-6} \mathrm{~cm} / \mathrm{s}$. In case with $\sigma=\infty$ model is terminated with $n=m=6$. When the analysis is extended to higher harmonics, the flow pattern is supposed to be more small-scale predominant.

It is surprising that such slow velocity is capable of producing the observed drifting field. It is supposed to be caused by such extreme conductivity as infinite conductivity assigned to the generating layer Region II. Much higher velocity is expected for the finite conductivity.

\section{Finite electrical conductivity in Region II}

Following the procedures of the infinite conductivity case, we have extended our study to a finite conductivity model. In this section, we summarize the results. Since the observed type of sectorial poloidal field is produced only by toroidal flow $\psi$ and the whole process is essentially the same as that of the infinite conductivity case, we describe the results for toroidal flow $\psi$.

\section{Magnetic potential}

For finite conductivity, $\eta^{\prime} \neq 0$. Then, the induction Eqs. (17) and (18) become impossible to solve analytically. Therefore, we were obliged to resort to a crude approximation. Let $\varepsilon$ be

$$
\varepsilon=\frac{1}{r_{0}} \frac{r_{0}+r_{1}}{2}
$$

We solve the following equations for toroidal and poloidal magnetic potentials induced by toroidal flow;

$$
\left[\frac{\partial}{\partial t}-\eta^{\prime} \nabla^{2}\right] \frac{L^{2} T}{r^{\prime}}=\frac{B_{0}}{\varepsilon^{5} r_{0}}\left[2 D_{A} \frac{\partial}{\partial r^{\prime}}\left(r^{\prime} \frac{\psi}{r^{\prime 3}}\right)+D_{B} L^{2}\left(\frac{\psi}{r^{\prime 3}}\right)\right],
$$

$$
\left[\frac{\partial}{\partial t}-\eta^{\prime} \nabla^{2}\right] \frac{L^{2} S}{r^{\prime}}=\frac{2 B_{0}}{\varepsilon^{4}} \frac{\partial \psi}{\partial \varphi} .
$$

Then, we obtain

$$
\begin{gathered}
T=\sum_{n} \sum_{m}\left[\frac { i B _ { 0 } } { \varepsilon ^ { 5 } r _ { 0 } } \left\{\frac{1}{\omega-i \omega_{\eta, n+1}} a_{n+1}^{m} t_{u, n}^{m} r^{\prime} \tilde{G}_{u, n+1}\left(\alpha_{n+1} r^{\prime}\right)\right.\right. \\
\left.-\frac{1}{\omega-i \omega_{\eta, n-1}} a_{n-1}^{m} t_{v, n}^{m} r^{\prime} \tilde{G}_{v, n-1}\left(\alpha_{n-1} r^{\prime}\right)\right\} \\
\left.+\frac{r^{\prime}}{n(n+1)}\left\{A_{t, n}^{m} j_{n}\left(k^{\prime} r^{\prime}\right)+B_{t, n}^{m} n_{n}\left(k^{\prime} r^{\prime}\right)\right\}\right] P_{n}^{m}(\cos \theta) e^{i m \varphi} e^{i \omega t} \\
S=\sum_{n} \sum_{m}\left[\frac{2 B_{0}}{\varepsilon^{4}} \frac{1}{\omega-i \omega_{\eta, n}} a_{n}^{m} s_{n}^{m} r^{\prime} G_{n}\left(\alpha_{n} r^{\prime}\right)\right. \\
\left.\quad+\frac{r^{\prime}}{n(n+1)}\left\{A_{s, n}^{m} j_{n}\left(k^{\prime} r^{\prime}\right)+B_{s, n}^{m} n_{n}\left(k^{\prime} r^{\prime}\right)\right\}\right] \\
P_{n}^{m}(\cos \theta) e^{i m \varphi} e^{i \omega t}
\end{gathered}
$$


where

$$
\left.\begin{array}{c}
k^{\prime 2}=-\frac{i \omega}{\eta^{\prime}} \\
t_{u, n}^{m}=\frac{(n+2)(n+m+1)}{(n+1)(2 n+3)}, \quad t_{v, n}^{m}=\frac{(n-1)(n-m)}{n(2 n-1)}, \\
s_{n}^{m}=\frac{m}{n(n+1)}, \\
\tilde{G}_{u, n+1}\left(\alpha_{n+1} r^{\prime}\right)=3(n+3) G_{n+1}\left(\alpha_{n+1} r^{\prime}\right)-2 \alpha_{n+1} \varepsilon G_{n}\left(\alpha_{n+1} r^{\prime}\right) \\
\tilde{G}_{v, n-1}\left(\alpha_{n-1} r^{\prime}\right)=3(n-2) G_{n-1}\left(\alpha_{n-1} r^{\prime}\right)-2 \alpha_{n-1} \varepsilon G_{n}\left(\alpha_{n-1} r^{\prime}\right)
\end{array}\right\}
$$

For $A_{t, n}^{m}, B_{t, n}^{m}, A_{s, n}^{m}$, and $B_{s, n}^{m}$, see "Appendix: Coefficients in Eqs. (50), (51)."

Since the normal component of the electric current must vanish at the core-mantle boundary, $T$ must be zero at the boundary. As the normal component of the current, here we take that of the current due to the induction before the current diffuses, which means that we take the current produced by the first term of (50) with the second term set aside. Then, we obtain,

$$
\begin{aligned}
& a_{n+1}^{m}=a_{n-1}^{m} r_{n}^{m}, \\
& r_{n}^{m}=\tilde{r}_{n}^{m} \frac{\omega-i \omega_{\eta, n+1}}{\omega-i \omega_{\eta, n-1}} \\
& \tilde{r}_{n}^{m}=\frac{\mathrm{g} v\left(r_{0}^{\prime}\right)}{\mathrm{g} u\left(r_{0}^{\prime}\right)} \\
& \left.\mathrm{g} u\left(r_{0}^{\prime}\right)=n(n+1) t_{u, n}^{m} \tilde{G}_{u, n+1}\left(\alpha_{n+1} r^{\prime}\right)\right\} . \\
& \left.\mathrm{g} v\left(r_{0}^{\prime}\right)=n(n+1) t_{v, n}^{m} \tilde{G}_{u, n-1}\left(\alpha_{n-1} r^{\prime}\right)\right\} \text {. }
\end{aligned}
$$

It follows that

$$
\begin{aligned}
& a_{m+2 j-1}^{m}=0, \\
& \frac{a_{m+2 j}^{m}}{a_{m+2 j-2}^{m}}=r_{m+2 j-1}^{m}=\tilde{r}_{m+2 j-1}^{m} \frac{\omega-i \omega_{\eta, m+2 j}}{\omega-i \omega_{\eta, m+2 j-2}} \\
& \quad(j=1,2,3, \ldots) .
\end{aligned}
$$

This means that the toroidal velocity potential $\psi$ is a function symmetric about the equator. Then, $a_{m+2 j}^{m}$ is derived from $a_{m}^{m}$ by the equation
Table 3 Relative amplitude $a_{n}^{m}$ for $r_{1}^{\prime}=0.9$ and $i=1$

\begin{tabular}{lllll}
\hline & $\boldsymbol{a}_{\boldsymbol{m}}^{\boldsymbol{m}}$ & $\boldsymbol{a}_{\boldsymbol{m}+\mathbf{2}}^{\boldsymbol{m}}$ & $\boldsymbol{a}_{\boldsymbol{m}+\mathbf{4}}^{\boldsymbol{m}}$ & $\boldsymbol{a}_{\boldsymbol{m}+\mathbf{6}}^{\boldsymbol{m}}$ \\
\hline$m=2$ & 1 & $3.3 \times 10^{-2}$ & $5.3 \times 10^{-3}$ & $1.4 \times 10^{-3}$ \\
$m=3$ & 1 & $4.3 \times 10^{-2}$ & $6.2 \times 10^{-3}$ & $1.4 \times 10^{-3}$ \\
$m=4$ & 1 & $4.3 \times 10^{-2}$ & $5.6 \times 10^{-3}$ & $1.1 \times 10^{-3}$ \\
$m=5$ & 1 & $4.0 \times 10^{-2}$ & $4.7 \times 10^{-3}$ & $8.3 \times 10^{-4}$ \\
$m=6$ & 1 & $3.7 \times 10^{-2}$ & $3.9 \times 10^{-3}$ & $6.2 \times 10^{-4}$ \\
\hline
\end{tabular}

Finite conductivity model

$$
\frac{a_{m+2 j \max }^{m}}{a_{m}^{m}}=\prod_{j=1}^{j \max } r_{m+2 j-1}^{m}=\frac{\omega-i \omega_{\eta, m+2 j \max }}{\omega-i \omega_{\eta, m}} \prod_{j=1}^{j \max } \tilde{r}_{m+2 j-1}^{m} .
$$

At this stage, $\omega$ is unknown. Approximating $\omega_{\eta, m+2 j}$ and $\omega_{\eta, m+2 j-2}$ by $\omega_{\eta, m+2 j-1}$, we have

$$
\frac{a_{m+2 j \max }^{m}}{a_{m}^{m}}=\prod_{j=1}^{j \max } \tilde{r}_{m+2 j-1}^{m}
$$

Then, similarly to the infinite conductivity case, it has become clear that the sectorial term $\left(a_{m}^{m}\right)$ is by far larger than any other terms $\left(a_{m+2 j}^{m}\right)$ as shown in Table 3 . The results are essentially the same as those for the infinite conductivity. The difference between the results is due to the approximation in the finite conductivity case as in (48) and (49).

Since the odd coefficients of the velocity potential are zero $\left[a_{m+2 j-1}^{m}=0\right.$ in (53.1)], the poloidal magnetic potential $S$ becomes symmetric about the equator, while the toroidal potential $T$ is anti-symmetric. This is clearly seen in the following equations.

$$
\begin{aligned}
S= & \frac{2 B_{0}}{\varepsilon^{4}} \sum_{m} \sum_{j=0} \sum_{i} \frac{1}{\omega-i \omega_{\eta, m+2 j, i}} a_{m+2 j, i}^{m} s_{m+2 j}^{m} \\
& \times\left[r^{\prime} G_{m+2 j}\left(\alpha_{m+2 j, i} r^{\prime}\right)+r^{\prime}\left\{\bar{A}_{s, m+2 j, i}^{m} \bar{j}_{m+2 j}\left(k^{\prime} r^{\prime}\right)\right.\right. \\
& \left.\left.+\bar{B}_{s, m+2 j, i}^{m} \bar{n}_{m+2 j}\left(k^{\prime} r^{\prime}\right)\right\}\right] P_{m+2 j}^{m}(\cos \theta) e^{i m \varphi} e^{i \omega t} \\
& s_{m+2 j}^{m}=\frac{m}{(m+2 j)(m+2 j+1)},
\end{aligned}
$$

$$
\begin{aligned}
T= & \frac{i B_{0}}{\varepsilon^{5} r_{0}} \sum_{m} \sum_{j=0} \sum_{i}\left[a _ { m + 2 j , i } ^ { m } \frac { 1 } { \omega - i \omega _ { \eta , m + 2 j , i } } \left\{t_{u, m+2 j-1}^{m} r^{\prime} \tilde{G}_{u, m+2 j}\left(\alpha_{m+2 j} r^{\prime}\right) P_{m+2 j-1}^{m}(\cos \theta)\right.\right. \\
& \left.-t_{u, m+2 j+1}^{m} r^{\prime} \tilde{G}_{u, m+2 j}\left(\alpha_{m+2 j} r^{\prime}\right) P_{m+2 j+1}^{m}(\cos \theta)\right\}+a_{m+2 j, i}^{m} \frac{r^{\prime}}{(m+2 j+1)(m+2 j+2)}\left\{\bar{A}_{t, m+2 j+1}^{m} \bar{j}_{m+2 j+1}\left(k^{\prime} r^{\prime}\right)\right. \\
& \left.\left.+\bar{B}_{t, m+2 j+1}^{m} \bar{n}_{m+2 j+1}\left(k^{\prime} r^{\prime}\right)\right\} P_{m+2 j+1}^{m}(\cos \theta)\right] e^{i m \varphi} e^{i \omega t}
\end{aligned}
$$




$$
\left.\begin{array}{c}
t_{u, m+2 j-1}^{m}=\frac{2(m+2 j+1)(m+j)}{(m+2 j)(2 m+4 j+1)}, \\
t_{v, m+2 j+2}^{m}=\frac{(m+2 j)(2 j+1)}{(m+2 j+1)(2 m+4 j+1)}, \\
\tilde{G}_{u, m+2 j}\left(\alpha_{m+2 j} r^{\prime}\right)=3(m+2 j+2) G_{m+2 j}\left(\alpha_{m+2 j} r^{\prime}\right) \\
-2 \alpha_{m+2 j-1} \varepsilon G_{m+2 j-1}\left(\alpha_{m+2 j} r^{\prime}\right) \\
\tilde{G}_{v, m+2 j}\left(\alpha_{m+2 j} r^{\prime}\right)=3(m+2 j-1) G_{m+2 j}\left(\alpha_{m+2 j} r^{\prime}\right) \\
-2 \alpha_{m+2 j-1} \varepsilon G_{m+2 j+1}\left(\alpha_{m+2 j} r^{\prime}\right)
\end{array}\right\} .
$$

In the case of infinite conductivity, predominance of the sectorial terms of velocity $a_{m}^{m}$ has led to the predominance of sectorial terms in $S$, as in the observed drifting field. In order to confirm whether this is also true in the finite conductivity case, we need to estimate the frequency $\omega$.

\section{Characteristic frequency}

Following the procedure as in the case for the infinite conductivity case, we calculate representative frequencies. Corresponding to Eq. (40), we obtain

$$
\begin{aligned}
& \left\{n(n+1) \omega m-2 m \Omega_{0}\right\} \int_{r_{1}^{\prime}}^{1}\left[G_{n}\left(\alpha_{n} r^{\prime}\right)\right]^{2} r^{\prime 2} \mathrm{~d} r^{\prime} \\
& \quad+\frac{\Omega_{B}^{2}}{\omega-i \omega_{\eta, n}} \int_{r_{1}^{\prime}}^{1} \frac{1}{r^{\prime 4}}\left\{U_{n}^{m}+V_{n}^{m}+W_{n}^{m}\right\} G_{n}\left(\alpha_{n} r^{\prime}\right) r^{\prime 2} \mathrm{~d} r^{\prime}=0 .
\end{aligned}
$$

Unfortunately, this is intractable, because $V_{n}^{m}$ and $W_{n}^{m}$ contain $\omega$. (For $U_{n}^{m}, V_{n}^{m}$, and $W_{n}^{m}$, see "Appendix: Coefficients in Eqs. (57), (58).") However, if we assign an approximate value to $\omega$ in $V_{n}^{m}$ and $W_{n}^{m}$, we can solve the equation. Then, we obtain the frequencies,

$$
\left.\begin{array}{rl}
\omega_{m+2 j}^{i} & =\frac{2 m}{(m+2 j)(m+2 j+1)} \Omega_{0} \\
\omega_{m+2 j} & =\frac{1}{2 m} \frac{\Omega_{B}^{2}}{\Omega_{0}} \frac{1}{\varepsilon^{5} g g_{m+2 j}} q_{w, m+2 j}+i \omega_{\eta, m+2 j}
\end{array}\right\}
$$

where $\omega_{m+2 j}^{i}$ is the inertial frequency and $\omega_{m+2 j}$ is the magnetostrophic frequency. For $\operatorname{gg}_{m+2 j}$ and $q_{w, m+2 j}$, see also "Appendix: Coefficients in Eqs. (57), (58)." Here, we employ an iterative procedure. Giving an initial guess $\omega_{0}$ to $\omega$ in $V_{n}^{m}$ and $W_{n}^{m}$, we calculate $\omega_{m}$. Adopting the obtained $\omega_{m}$ as the initial guess in the next step, we repeat the process. $\omega_{m}$ obtained in this way is shown in Table 4. Although the convergence is not necessarily good, here we adopt the Step 3. Then, the frequencies are obtained as in Table 5. They are higher than in the case of infinite conductivity.
Table 4 Characteristic magnetostrophic frequency $\omega_{m}$ $\left(s^{-1}\right)$ for $m=2, r_{1}^{\prime}=0.9$ and $i=1$

\begin{tabular}{lll}
\hline & Initial guess $\boldsymbol{\omega}_{\mathbf{2}}\left(\mathbf{s}^{\mathbf{- 1}}\right)$ & Obtained $\left.\boldsymbol{\omega}_{\mathbf{2}} \mathbf{( s}^{-\mathbf{1}}\right)$ \\
\hline Step 1 & $2.83 \times 10^{-12}+1.31 \times 10^{-13} ;$ & $6.41 \times 10^{-13}+1.02 \times 10^{-12} ;$ \\
Step 2 & $6.41 \times 10^{-12}+1.02 \times 10^{-12} ;$ & $3.67 \times 10^{-13}+7.26 \times 10^{-12} ;$ \\
Step 3 & $3.67 \times 10^{-13}+7.26 \times 10^{-12} ;$ & $4.18 \times 10^{-13}+6.55 \times 10^{-12} ;$ \\
\hline
\end{tabular}

Finite conductivity model

Table 5 Characteristic magnetostrophic frequency $\omega_{m}$ $\left(s^{-1}\right)$ for $r_{1}^{\prime}=0.9$ and $i=1$

\begin{tabular}{ll}
\hline & $\boldsymbol{\omega}_{m}^{m}\left(\mathbf{s}^{-\mathbf{1}}\right)$ \\
\hline$m=2$ & $4.18 \times 10^{-13}+6.55 \times 10^{-12} ;$ \\
$m=3$ & $1.25 \times 10^{-12}+1.62 \times 10^{-12} ;$ \\
$m=4$ & $2.48 \times 10^{-12}+2.82 \times 10^{-12} ;$ \\
$m=5$ & $4.09 \times 10^{-12}+4.17 \times 10^{-12} ;$ \\
$m=6$ & $6.06 \times 10^{-12}+5.69 \times 10^{-12} ;$ \\
\hline
\end{tabular}

Finite conductivity model

\section{Predominance of sectorial terms in the toroidal flow and the poloidal field}

Now, the frequency has been obtained, and we can calculate the magnetic potential $S$ and $T$. We rewrite (55) and (56) as follows.

$$
\begin{aligned}
S= & \frac{2 B_{0}}{\varepsilon^{4}} \sum_{n} \sum_{m}\left[S_{g, n}^{m} r^{\prime} G_{n}\left(\alpha_{n} r^{\prime}\right)+r^{\prime}\left\{S_{A, n, j}^{m} \bar{j}_{n}\left(k_{n}^{\prime} r^{\prime}\right)\right.\right. \\
& \left.\left.+S_{B, n, i}^{m} \bar{n}_{n}\left(k_{n}^{\prime} r^{\prime}\right)\right\}\right] P_{n}^{m}(\cos \theta) e^{i m \varphi} e^{i \omega t},
\end{aligned}
$$

$$
\begin{aligned}
T= & \frac{i B_{0}}{\varepsilon^{5} r_{0}} \sum_{n} \sum_{m}\left[\left\{T_{g u, n}^{m} r^{\prime} \tilde{G}_{u, n+1}\left(\alpha_{n+1} r^{\prime}\right)-T_{g v, n}^{m} r^{\prime} \tilde{G}_{v, n-1}\left(\alpha_{n-1} r^{\prime}\right)\right\}\right. \\
& \left.-\left\{T_{A, n}^{m} r^{\prime} \bar{j}_{n}\left(k_{n}^{\prime} r^{\prime}\right)+T_{B, n}^{m} r^{\prime} \bar{n}_{n}\left(k_{n}^{\prime} r^{\prime}\right)\right\}\right] P_{n}^{m}(\cos \theta) e^{i m \varphi} e^{i \omega t}, \quad(60)
\end{aligned}
$$

where

$$
\begin{aligned}
S_{g, n}^{m} & =\frac{1}{\omega-i \omega_{\eta, n}} a_{n}^{m} s_{n}^{m}, S_{A, n}^{m}=\frac{1}{\omega-i \omega_{\eta, n}} a_{n}^{m} s_{n}^{m} \bar{A}_{s, n}^{m}, \\
S_{B, n}^{m} & =\frac{1}{\omega-i \omega_{\eta, n}} a_{n}^{m} s_{n}^{m} \bar{B}_{s, n}^{m}, \\
T_{g u, n}^{m} & =\frac{1}{\omega-i \omega_{\eta, n+1}} a_{n+1}^{m} t_{u, n}^{m}, \quad T_{g v, n}^{m}=\frac{1}{\omega-i \omega_{\eta, n-1}} a_{n-1}^{m} t_{v, n}^{m}, \\
T_{A, n}^{m} & =\frac{1}{\omega-i \omega_{\eta, n-1}} a_{n-1}^{m} \frac{1}{n(n+1)} \bar{A}_{t, n}^{m}, \\
T_{B, n}^{m} & =\frac{1}{\omega-i \omega_{\eta, n-1}} a_{n-1}^{m} \frac{1}{n(n+1)} \bar{B}_{t, n}^{m},
\end{aligned}
$$



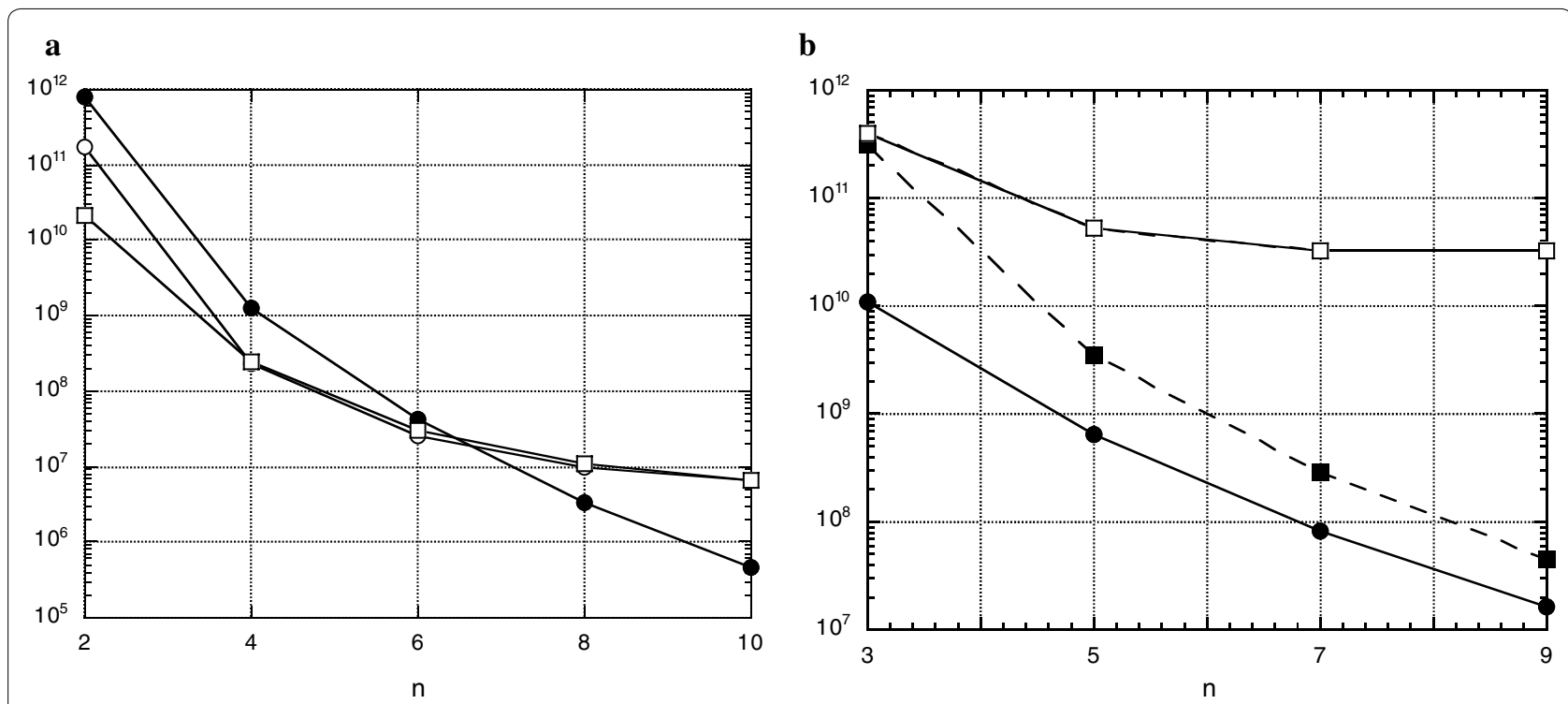

Fig. 5 Predominance of a sectorial harmonics in poloidal field and $\mathbf{b}$ of $n=m+1$ term in toroidal field. a Solid circle, $\left|S_{g, n, i}^{m}\right|:$ open circle, $\left|S_{A, n, i}^{m}\right|:$ open square, $\left|S_{B, n, i}^{m}\right|$, b solid circle, $\left|T_{g u, n, i}^{m}\right|:$ solid square, $\left|T_{g v, n, i}^{m}\right|$ : open circle, $\left|T_{A, n, i}^{m}\right|:$ Open square, $\left|T_{B, n, i}^{m}\right| \cdot\left|T_{A, n, i}^{m}\right|$ (open circle) is almost the same as $\left|T_{B, n, i}^{m}\right|$ (open square)

Then, we obtain $S_{g, n}^{m}, S_{A, n}^{m}$ and $S_{B, n}^{m}$. Taking $a_{n}^{m}=1$ their absolute values are plotted in Fig. $5 \mathrm{a}$ for $m=2$. The figure indicates that the sectorial harmonics $n=m$ dominate over other harmonics $n=m+2, m+4, \ldots$. This means that the observable field becomes sectorial term predominant. (In "Appendix: Explicit expression of $S$ in (59) for $m=2$ ", $S$ for $m=2$ is given with numerical coefficients.)

Absolute values of $\left\{T_{g u, n}^{m}, T_{g v, n}^{m}, T_{A, n}^{m}, T_{B, n}^{m}\right\}$ are plotted

in Fig. $5 \mathrm{~b}$ for $m=2$. In this case, the term for $n=m+1$ is predominant. The field is anti-symmetric about the equator.

\section{Toroidal velocity}

Similarly to the infinite conductivity case, we can estimate the toroidal velocity that induces the observed drifting field. The flow pattern compatible with the observed drifting field is shown in Fig. 6. In the figure, shown is the flow responsible only for inducing the sectorial part of the drifting field. When compared with that for the infinite conductivity model in Fig. 3, it is clear that the pattern is dominated by lower harmonics such as $m=2$, while in the infinite conductivity model, higher harmonic flow is outstanding.

The maximum velocity at the equator at the core surface is plotted against order $m$ in Fig. 7. A difference from the case of infinite conductivity is that the velocity for

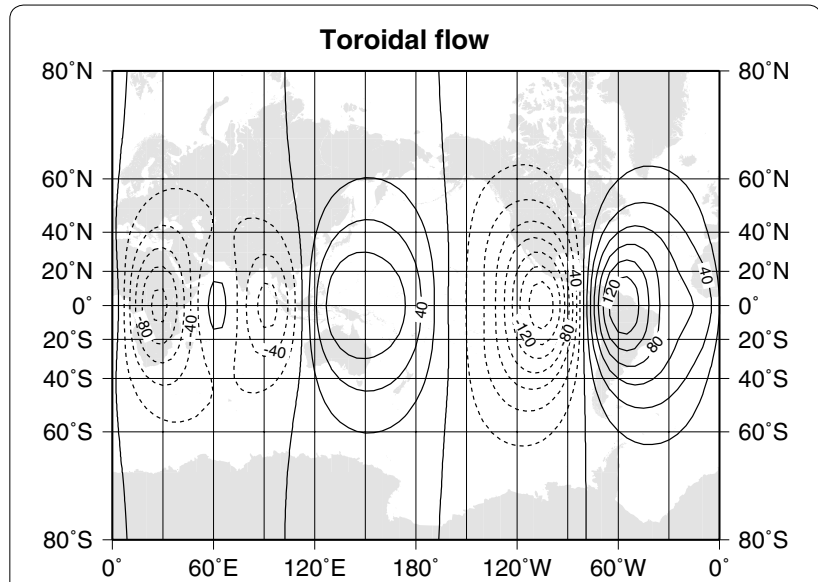

Fig. 6 Velocity potential $\psi$ at the core surface estimated from the drifting field at year 2000 AD. Unit is $10^{-6} \mathrm{~cm} / \mathrm{s}$. Assumed electrical conductivity of the core is $\sigma=10^{5} \mathrm{~S} / \mathrm{m}$

$m=2$ is faster than any other harmonics. For $m=2$, the velocity is $1.7 \times 10^{-4} \mathrm{~cm} / \mathrm{s}$, while it is $1.4 \times 10^{-4} \mathrm{~cm} / \mathrm{s}$ for $m=6$. A further difference is that the velocity is $10-10^{2}$ times faster than that of the infinite conductivity case. This is reasonable because, in the finite conductivity case, greater energy is required for the liquid flow against Joule dissipation to generate the observed field. 


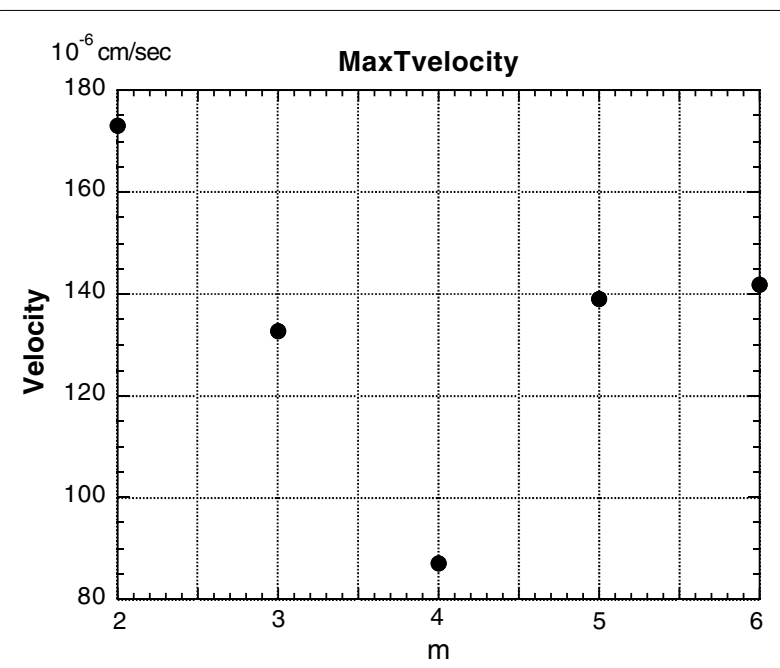

Fig. 7 Maximum velocity at the equator on the core surface. In the unit of $10^{-6} \mathrm{~cm} / \mathrm{s}$. In case $\sigma=10^{5} \mathrm{~S} / \mathrm{m}$

\section{Summary of the results}

It has been presumed that the observed drifting field is produced by interaction of core surface flow with the axial dipole field. In order to understand the essential process, we have examined an extreme case. The mantle is electrically insulating, and the field-generating layer is of infinite conductivity. Fluid viscosity and thermal diffusivity are zero. Then, the top surface is stress free. Although the observed field is not a simple dipole field but the nondipole field also exists near the core-mantle boundary, we approximated the field by an axial dipole field only. Then, we examined the field generated by fluid flows in a thin shell at the core surface.

We have found that the boundary condition on the electric current at the core-mantle boundary exerts the vital effect on generating the observed type of drifting field. The normal component of the current must vanish at the core-mantle boundary. This strongly constrains the flow pattern at the surface. When the current induced by the toroidal flow satisfies the boundary condition, the flow becomes the type of sectorial harmonics predominant. Interacting with the dipole field, this flow induces the poloidal magnetic field with the sectorial harmonics predominant, which is exactly the same type of field as the observed drifting field. On the other hand, the poloidal flow becomes the flow confined on the meridional plane. It induces the zonal poloidal field that cannot be observed as the drifting field.

Figure 8 shows an example of the predominance of the sectorial term in the toroidal velocity potential $\psi$, which is $a_{n}^{2} / a_{2}^{2}$ for the infinite conductivity case. Even if the conductivity of the generating layer is finite such as

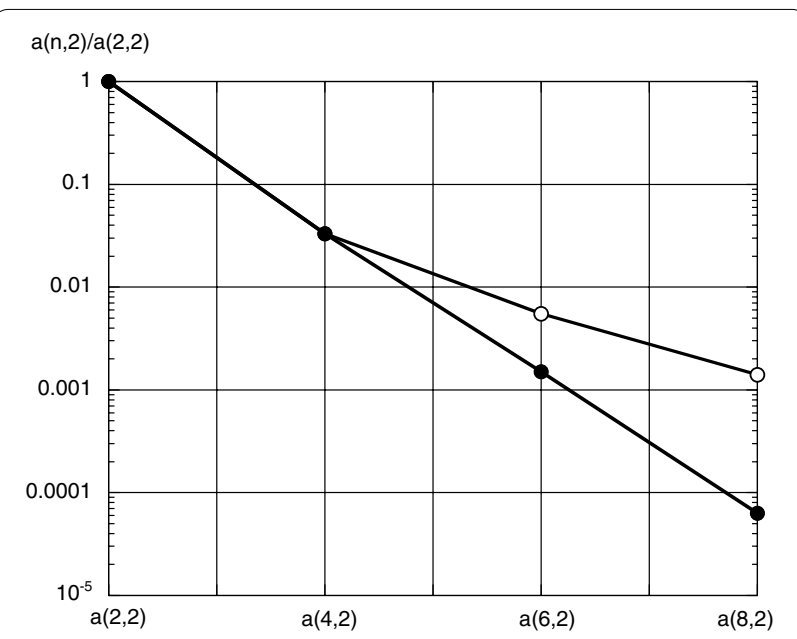

Fig. 8 Amplitude ratio $a_{n}^{2} / a_{2}^{2} \mathrm{~S} / \mathrm{m}$. Solid circle shows the case with $\sigma=\infty$ and open circle shows that with $\sigma=10^{5} \mathrm{~S} / \mathrm{m}$

$5 \times 10^{5} \mathrm{~S} / \mathrm{m}$, predominance of the sectorial harmonics still holds. It is noted that the coefficients $a_{n}^{m}$ are uniquely determined by the sectorial coefficient $a_{m}^{m}$, but $a_{m}^{m}$ is left undetermined. In order to fix $a_{m}^{m}$, some more additional conditions are needed.

The characteristic frequency is different between the two models. For the infinite conductivity, the frequency is lower than for the finite conductivity. It is $\omega_{m}=-8.8 \times 10^{-14} 1 / \mathrm{s}$ for $m=2$ for the infinite conductivity, whereas it is $\omega_{m}=4.2 \times 10^{-13}+6.6 \times 10^{-13} i 1 / \mathrm{s}$ for $m=2$ for the finite conductivity model. Since the toroidal velocity is proportional to frequency, the velocity to induce the observed magnitude of the drifting field is slower for the infinite conductivity than for the finite conductivity. It is about $10^{-6}-10^{-5} \mathrm{~cm} / \mathrm{s}$ for the infinite conductivity, while it is about $10^{-4} \mathrm{~cm} / \mathrm{s}$ for the finite conductivity. It is reasonable that the slower velocity is sufficient for the higher conductivity to induce the observed field. It is also noted that the velocity obtained here is much slower than the velocity inferred from the westward drift of the magnetic field, $10^{-2} \mathrm{~cm} / \mathrm{s}$.

It is unknown how thick the surface flow is that effectively contributes to the generation of the drifting field. Changing the thickness of the surface layer, we have examined the change of frequency for the infinite conductivity model. Table 6 shows the characteristic frequency for the thickness $(D)$ of $175 \mathrm{~km}, 350 \mathrm{~km}$ and $700 \mathrm{~km}$ in case of infinite conductivity. The frequency increases with the thickness. This may sound somewhat strange, because the magnetostrophic frequency decreases with thickness as $D^{-2}$, provided the magnetic field is constant. In the present case, however, the dipole field increases its intensity with depth as $r^{-3}$. This intensity change is supposed to affect the frequency increase 
Table 6 Characteristic frequency $\omega_{m}\left(\mathrm{~s}^{-1}\right)$ for $i=1$

\begin{tabular}{llll}
\hline & $\boldsymbol{D}=\mathbf{1 7 5}(\mathbf{k m})$ & $\boldsymbol{D}=\mathbf{3 5 0}(\mathbf{k m})$ & $\boldsymbol{D}=\mathbf{7 0 0}(\mathbf{k m})$ \\
\hline$m=2$ & $-7.1 \times 10^{-14}$ & $-8.8 \times 10^{-14}$ & $-1.4 \times 10^{-13}$ \\
$m=3$ & $-5.9 \times 10^{-13}$ & $-7.3 \times 10^{-13}$ & $-1.1 \times 10^{-12}$ \\
$m=4$ & $-1.5 \times 10^{-12}$ & $-1.9 \times 10^{-12}$ & $-2.9 \times 10^{-12}$ \\
\hline
\end{tabular}

Infinite conductivity model

with thickness. As discussed before, the increase in frequency leads to increase in the fluid velocity that is needed to produce the observed drifting field. However, the frequency change is not so large as in the case of changing conductivity. Therefore, the change in the velocity due to the change of the thickness is rather limited. This suggests that participation of the deeper part of the surface flow is not very sensitive in generating the drifting field.

Inviscid fluid with zero viscosity is another extreme approximation. This allows the free surface condition at the core-mantle boundary. However, this is not an actual case. Since the fluid core is viscous liquid, the core-mantle boundary must be taken as the rigid surface. Surprisingly if the rigid boundary condition is applied, the radial component of the field induced by fluid flow becomes zero at the core-mantle boundary, so that the induced field cannot be observed at the Earth's surface. This problem will be surmounted by attaching a thin viscous layer with finite electrical conductivity to the mantle. Then, the boundary between this thin layer and the field-generating layer is stress-free surface. The thin top layer works only to attenuate the field from inside.

\section{Discussion}

In this study, we have pursued a generation process of the drifting field, considering that the drifting and standing fields are of different origin. This is a different approach to the core dynamics from that of many current investigations that attempt to derive the flow structure from the total field without discriminating the different type of fields. Such remarkable features as westward drifting flux patches in the equatorial region of the Atlantic hemisphere or low secular variation in the Pacific area are common issues to these investigations. They were attempted to explain by specific numerical dynamo simulations by ascribing inhomogeneous boundary conditions at the inner-outer core or core-mantle boundary (e.g., Amit and Choblet 2012; Aubert et al. 2013).

In our model, however, these features are a natural consequence of coexistence of two types of fields, drifting and standing fields (Yukutake and Shimizu 2015, 2016). Westward drift becomes remarkable at the equator by superposition of the standing field, whose vertical component is anti-symmetric about the equator, on the sectorial type of equator-symmetric drifting field. Time variation is also different between the two types of fields. Some of the standing fields are growing rapidly, and the other decaying. Low secular variation in the Pacific is supposed to be caused by the drifting field's passing over the changing standing fields. The problem is, therefore, to find the flow structure of the drifting and the standing fields separately.

One of the attractive ideas about the westward drift is wave propagation. Magnetostrophic wave (often called magnetic Rossby wave) generated by combined effect of Coriolis, Lorentz and/or gravitational forces has been considered to be a cause of the westward drift of the magnetic field (see, e.g., Finlay et al. 2010 for review). Yoshida and Hamano (1993), Hori et al. (2018), for example, examined the magnetostrophic waves in the framework of quasi-geostrophic approximation. Yoshida and Hamano (1993) obtained the westward drifting field with phase velocity similar to the observed, which was excited by the oscillation of the mantle with respect to the core with heterogeneous topographic bottom boundary. To be noted is that they obtained the wave that was symmetric about the equator as expressed by sectorial harmonics. That is exactly the type of the observed field. This stems from the type of the toroidal field they assumed as the background. It was equatorially symmetric field. Interaction of the columnar flow, which is essentially symmetric type in the quasi-geostrophic approximation, with the symmetric toroidal field induces equatorially symmetric poloidal field. Accordingly, the problem in their study is whether the toroidal field is equatorially symmetric or not. Hori et al. (2018) conducted geodynamo simulations and found the magnetostrophic waves that were compatible with those derived from quasi-geostrophic approximation. Unfortunately, however, their waves were of equatorially anti-symmetric type. This is due to the toroidal field in their simulation, which was anti-symmetric about the equator, although it is very usual in the geodynamo simulations (see, e.g., Christensen and Wicht 2009). Through interaction with anti-symmetric toroidal field, columnar flow induces anti-symmetric field.

Common to these wave theories there is a serious problem. It is a dispersive nature of the wave velocity. The phase velocity highly depends on the harmonics, while there is no significant dispersive relationship between harmonics in the observed field (Yukutake and Shimizu 2015). Considering the nondispersive drift velocity, we considered in this study that the field is buried in the zonal flow near the core surface and carried westwards as a whole. The zonal flow has been approximated by a rotating spherical shell. This is similar to the classical Bullard et al's model (1950). Since this is a very crude 
approximation and the change of the layer thickness does not have serious effect on the results, it does not necessarily rule out the possibility of the flow structure gradually changing with depth. However, the approximation by the surface layer is in harmony with the stratification model near the core surface (e.g., Braginsky 1998, 1999; Buffet 2014). And it seems to favor the stratification model.

Regarding the sectorial nature of the drifting field, we have considered interaction of sectorial toroidal flow with the dipole field. This interaction induces the observed type of sectorial poloidal field. The problem is why the surface toroidal flow is sectorial. This has been solved by the boundary condition on the electric current. Into the insulating mantle, no electric current is allowed to penetrate. This condition strongly constrains the surface flow. Then, it has been found that only the sectorial type flows are possible to exist.

Regarding the standing field, we have not deepened the examination. We simply guess, as in many of dynamo simulations, that it is produced by columnar rolls in the deeper part of the core under the surface layer. Interacting with the anti-symmetric toroidal field, the columnar flow induces anti-symmetric poloidal field. For example, the intense anomaly rapidly growing over the Eurasian continent is supposed to be one of those products.

\section{Conclusions}

The drifting field is comprised mostly of sectorial harmonics. This is one of the characteristic features of the drifting field. We pursued the cause of this characteristic feature. A simple model was employed.

The field is generated by fluid flow within the surface layer of thickness $D$ (e.g., $D=350 \mathrm{~km}$ ). The geomagnetic field near the core-mantle boundary is approximated by an axial dipole field. The core fluid is inviscid. The mantle is electrically insulating. Regarding the electrical conductivity of the generating layer, we examined two cases, infinite conductivity and finite conductivity $\left(5 \times 10^{5} \mathrm{~S} / \mathrm{m}\right)$.

The normal component of the electric current must vanish at the core-mantle boundary. This boundary condition imposes a strong constraint on the fluid motion in the layer. The toroidal flow that produces the current with no normal component at the boundary becomes sectorial type flow that consists of harmonics $P_{m+2 j}^{m}(\cos \theta)(j=0,1$, $2,3, \ldots)$ with sectorial harmonics $P_{m}^{m}(\cos \theta)$ predominant. The poloidal flow becomes meridional type flow that is expressed only by zonal harmonics $P_{n}^{0}(\cos \theta)$. The flow is confined on the meridional plane.

Interaction of the sectorial type toroidal flow with the axial dipole field induces the sectorial type poloidal field. Since the sectorial harmonics dominates in the toroidal flow, the sectorial harmonics is also predominant in the poloidal field. As the generating layer rotates westward, the sectorial field rotates together. This is observed as the drifting field with sectorial harmonics predominant. On the other hand, the meridional type flow, interacting with the axial dipole field, induces the poloidal field of zonal harmonics. Rotation of the zonal field induces zero secular variation. Therefore, the zonal field is not observed as the drifting field. Consequently, the sectorial type field produced by the sectorial type toroidal flow is the only field that is observed as the drifting field.

\section{Authors' contributions}

TY designed the study, carried out the calculations, and prepared the manuscript. HS helped in designing a part of research, checking the calculations, and preparing the manuscript. Both authors read and approved the final manuscript.

\section{Acknowledgements}

We thank two anonymous reviewers for their valuable comments, which were very helpful in improving the manuscript.

\section{Competing interests}

The authors declare that they have no competing interests.

\section{Availability of data and materials}

Drifting and standing magnetic field model is published as Table 1 in Yukutake and Shimizu (2015).

Funding

HS is supported by JSPS KAKENHI Grant Number $15 \mathrm{H} 05832$.

\section{Appendix: Explicit expression of $\boldsymbol{S}_{\boldsymbol{\psi}}$}

With $a_{m+2 j}^{m}$ in Table 1 Eq. (33) $S_{\psi}$ can be expressed as

$$
\begin{aligned}
S_{\psi}= & 2 B_{0} \frac{1}{r^{\prime 3}} a_{2}^{2}\left[\left\{0.33 G_{2}\left(\alpha_{2} r^{\prime}\right) P_{2}^{2}(\cos \theta)\right.\right. \\
& +3.3 \times 10^{-3} G_{4}\left(\alpha_{4} r^{\prime}\right) P_{4}^{2}(\cos \theta)+7.1 \times 10^{-5} G_{6}\left(\alpha_{6} r^{\prime}\right) P_{6}^{2}(\cos \theta) \\
& \left.+1.8 \times 10^{-6} G_{8}\left(\alpha_{8} r^{\prime}\right) P_{8}^{2}(\cos \theta)+\cdots\right\} \frac{1}{\omega_{2}} e^{2 i \varphi} e^{i \omega_{2} t} \\
& +a_{3}^{3}\left\{0.25 G_{3}\left(\alpha_{3} r^{\prime}\right) P_{3}^{3}(\cos \theta)+4.4 \times 10^{-3} G_{5}\left(\alpha_{5} r^{\prime}\right) P_{5}^{3}(\cos \theta)\right. \\
& +1.0 \times 10^{-4} G_{7}\left(\alpha_{7} r^{\prime}\right) P_{7}^{3}(\cos \theta) \\
& \left.\left.+2.5 \times 10^{-6} G_{9}\left(\alpha_{9} r^{\prime}\right) P_{9}^{3}(\cos \theta)+\cdots\right\} \frac{1}{\omega_{3}} e^{3 i \varphi} e^{i \omega_{3} t}+\cdots\right] .
\end{aligned}
$$




\section{Appendix: Coefficients in Eqs. (37) and (40)}

Coefficients in Eq. (37)

$$
\begin{aligned}
& \tilde{p}_{o, n}^{m}=\frac{\Omega_{B}^{2}}{\omega}\left\{\left(c_{t n 1}+\frac{4 m^{2}}{n(n+1)}\right) g_{n}+\left(c_{t n 2}+\frac{16 m^{2}}{n(n+1)}\right) d g_{n} \quad r_{t m 2}=-\frac{(n-1)(n-18)(n-m)}{n(2 n-1)}\right. \\
& \left.+\left(c_{t n 3}-\frac{4 m^{2}}{n(n+1)}\right) d_{2} g_{n}\right\} \\
& \tilde{p}_{a, n+2}^{m}=\frac{\Omega_{B}^{2}}{\omega}\left\{c_{t p 1} g_{n+2}+c_{t p 2} d g_{n+2}+c_{t p 3} d_{2} g_{n+2}\right\} \\
& \tilde{p}_{b, n-2}^{m}=\frac{\Omega_{B}^{2}}{\omega}\left\{c_{t m 1} g_{n-2}+c_{t m 2} d g_{n-2}+c_{t m 3} d_{2} g_{n-2}\right\} \\
& \tilde{p}_{c, n+1}^{m}=2 \Omega_{0} \frac{n(n+2)(n+m+1)}{2 n+3}\left\{d g g_{n+1}+(n+2) g g_{n+1}\right\} \\
& +\frac{2 \Omega_{B}^{2} m}{\omega}\left\{\left(r_{t p 0}+r_{s p 0}\right) g_{n+1}+\left(r_{t p 1}+r_{s p 1}\right) d g_{n+1}\right. \\
& \left.+\left(r_{t p 2}+r_{s p 2}\right) d_{2} g_{n+1}+\left(r_{t p 3}+r_{s p 3}\right) d_{3} g_{n+1}\right\} \\
& \tilde{p}_{d, n-1}^{m}=2 \Omega_{0} \frac{(n+1)(n-1)(n-m)}{2 n-1}\left\{d g g_{n-1}-(n-1) g g_{n-1}\right\} \\
& +\frac{2 \Omega_{B}^{2} m}{\omega}\left\{\left(r_{t m 0}+r_{s m 0}\right) g_{n-1}+\left(r_{t m 1}+r_{s m 1}\right) d g_{n-1}\right. \\
& \left.+\left(r_{t m 2}+r_{s m 2}\right) d_{2} g_{n-1}+\left(r_{t m 3}+r_{s m 3}\right) d_{3} g_{n-1}\right\} \\
& \left.\begin{array}{l}
c_{t m 1}=\frac{(n-2)(n+1)(n-m)(n-m+1)(n-6)(n-5)}{(2 n-3)(2 n-1)} \\
c_{t m 2}=\frac{2(n-2)(n+1)(n-m)(n-m+1)(2 n-9)}{(2 n-3)(2 n-1)} \\
c_{t m 3}=\frac{4(n-2)(n+1)(n-m)(n-m+1)}{(2 n-3)(2 n-1)}
\end{array}\right\} \\
& r_{s m 1}=-\frac{2(n-5)(n+1)(n+6)(n-m)}{n(2 n-1)} \\
& r_{t m 3}=-\frac{2(n-1)(n-m)}{n(2 n-1)} \\
& r_{t p 0}=\frac{(n+2)(n+5)\left(2 n^{2}+2 n+21\right)(n+m+1)}{(n+1)(2 n+3)} \\
& r_{t p 1}=-\frac{(n+2)\left(4 n^{2}+13 n+69\right)(n+m+1)}{(n+1)(2 n+3)} \\
& r_{t p 2}=\frac{(n+2)(n+19)(n+m+1)}{(n+1)(2 n+3)} \\
& r_{t p 3}=-\frac{2(n+2)(n+m+1)}{(n+1)(2 n+3)} \\
& r_{s m 0}=-\frac{(n-14)(n-3)(n+1)(n+2)(n-m)}{n(2 n-1)}
\end{aligned}
$$

\section{Coefficients in Eq. (40)}

$$
\left.\begin{array}{l}
c_{t n 1}=-\frac{(n-6)(n-1)(n+1)^{2}(n+4)(n-m)(n+m)}{n(2 n-1)(2 n+1)}-\frac{(n-3) n^{2}(n+2)(n+7)(n-m+1)(n+m+1)}{(n+1)(2 n+3)(2 n+1)} \\
c_{t n 2}=-\frac{16(n-1)(n+1)^{2}(n-m)(n+m)}{n(2 n-1)(2 n+1)}-\frac{16 n^{2}(n-m+1)(n+m+1)}{(n+1)(2 n+3)(2 n+1)} \\
c_{t n 2}=-\frac{4(n-1)(n+1)^{2}(n-m)(n+m)}{n(2 n-1)(2 n+1)}+\frac{4 n^{2}(n+2)(n-m+1)(n+m+1)}{(n+1)(2 n+3)(2 n+1)}
\end{array}\right\}
$$

$$
\begin{array}{lr}
c_{t p 1}=\frac{n(n+3)(n+m+1)(n+m+2)(n+6)(n+7)}{(2 n+3)(2 n+5)} \\
\left.\begin{array}{l}
c_{t p 2}=-\frac{2 n(n+3)(n+m+1)(n+m+2)(2 n+11)}{(2 n+3)(2 n+5)} \\
c_{t p 3}=\frac{4 n(n+3)(n+m+1)(n+m+2)}{(2 n+3)(2 n+5)}
\end{array}\right\} & r_{s m 2}=\frac{(n-18)(n+1)(n-m)}{n(2 n-1)} \\
r_{t m 0}=-\frac{(n-1)(n-4)\left(2 n^{2}+2 n+21\right)(n-m)}{n(2 n-1)} & r_{s m 3}=\frac{2(n+1)(n-m)}{n(2 n-1)}
\end{array}
$$




$$
\begin{aligned}
& r_{s p 0}=\frac{(n-1) n(n+4)(n+15)(n+m+1)}{(n+1)(2 n+3)} \\
& r_{s p 1}=-\frac{2(n-5) n(n+6)(n+m+1)}{(n+1)(2 n+3)} \\
& r_{s p 2}=-\frac{n(n+19)(n+m+1)}{(n+1)(2 n+3)} \\
& r_{s p 3}=\frac{2 n(n+m+1)}{(n+1)(2 n+3)} \\
& \operatorname{gg}_{n}=\int_{r_{1}}^{1} G_{n}\left(\alpha_{n} r\right) G_{n}\left(\alpha_{n} r\right) r^{2} \mathrm{~d} r \\
& \operatorname{gg}_{n \pm 1}=\int_{r_{1}}^{1} G_{n \pm 1}\left(\alpha_{n \pm 1} r\right) G_{n}\left(\alpha_{n} r\right) r^{2} \mathrm{~d} r \\
& d g g_{n}=\int_{r_{1}}^{1} \alpha_{n} r G_{n}^{\prime}\left(\alpha_{n} r\right) G_{n}\left(\alpha_{n} r\right) r^{2} \mathrm{~d} r \\
& =\int_{r_{1}}^{1} \frac{\mathrm{d} G_{n}\left(\alpha_{n} r\right)}{\mathrm{d} r} G_{n}\left(\alpha_{n} r\right) r^{3} \mathrm{~d} r \\
& d \operatorname{gg}_{n \pm 1}=\int_{r_{1}}^{1} \alpha_{n \pm 1} r G_{n \pm 1}^{\prime}\left(\alpha_{n \pm 1} r\right) G_{n}\left(\alpha_{n} r\right) r^{2} \mathrm{~d} r \\
& =\int_{r_{1}}^{1} \frac{\mathrm{d} G_{n \pm 1}\left(\alpha_{n \pm 1} r\right)}{\mathrm{d} r} G_{n}\left(\alpha_{n} r\right) r^{3} \mathrm{~d} r \\
& \mathrm{~g}_{n}=\int_{r_{1}}^{1} \frac{1}{r^{8}} G_{n}\left(\alpha_{n} r\right) G_{n}\left(\alpha_{n} r\right) r^{2} \mathrm{~d} r \\
& =\int_{r_{1}}^{1} \frac{1}{r^{6}} G_{n}\left(\alpha_{n} r\right) G_{n}\left(\alpha_{n} r\right) \mathrm{d} r \\
& \mathrm{~g}_{n \pm 2}=\int_{r_{1}}^{1} \frac{1}{r^{8}} G_{n \pm 2}\left(\alpha_{n \pm 2} r\right) G_{n}\left(\alpha_{n} r\right) r^{2} \mathrm{~d} r \\
& =\int_{r_{1}}^{1} \frac{1}{r^{6}} G_{n \pm 2}\left(\alpha_{n \pm 2} r\right) G_{n}\left(\alpha_{n} r\right) \mathrm{d} r
\end{aligned}
$$$$
\begin{aligned}
d g_{n} & =\int_{r_{1}}^{1} \frac{1}{r^{8}} \alpha_{n} r G_{n}^{\prime}\left(\alpha_{n} r\right) G_{n}\left(\alpha_{n} r\right) r^{2} \mathrm{~d} r \\
& =\int_{r_{1}}^{1} \frac{1}{r^{5}} \frac{d G_{n}\left(\alpha_{n} r\right)}{d r} G_{n}\left(\alpha_{n} r\right) \mathrm{d} r
\end{aligned}
$$$$
\frac{\mathrm{d} G_{n}\left(\alpha_{n} r\right)}{\mathrm{d} r}=\frac{\mathrm{d} z}{\mathrm{~d} r} \frac{\mathrm{d} G_{n}(z)}{\mathrm{d} z}=\alpha_{n} G_{n}^{\prime}\left(\alpha_{n} r\right)
$$$$
d g_{n \pm 1}=\int_{r_{1}}^{1} \frac{1}{r^{8}} \alpha_{n \pm 1} r G_{n \pm 1}^{\prime}\left(\alpha_{n \pm 1} r\right) G_{n}\left(\alpha_{n} r\right) r^{2} \mathrm{~d} r
$$$$
=\int_{r_{1}}^{1} \frac{1}{r^{5}} \frac{\mathrm{d} G_{n \pm 1}\left(\alpha_{n \pm 1} r\right)}{\mathrm{d} r} G_{n}\left(\alpha_{n} r\right) E r
$$$$
\operatorname{dg}_{n \pm 2}=\int_{r_{1}}^{1} \frac{1}{r^{8}} \alpha_{n \pm 2} r G_{n \pm 2}^{\prime}\left(\alpha_{n \pm 2} r\right) G_{n}\left(\alpha_{n} r\right) r^{2} \mathrm{~d} r
$$$$
=\int_{r_{1}}^{1} \frac{1}{r^{5}} \frac{\mathrm{d} G_{n \pm 2}\left(\alpha_{n \pm 2} r\right)}{\mathrm{d} r} G_{n}\left(\alpha_{n} r\right) \mathrm{d} r
$$$$
d_{2} g_{n}=\int_{r_{1}}^{1} \frac{1}{r^{8}}\left(\alpha_{n} r\right)^{2} G_{n}^{\prime \prime}\left(\alpha_{n} r\right) G_{n}\left(\alpha_{n} r\right) r^{2} \mathrm{~d} r
$$$$
=\int_{r_{1}}^{1} \frac{1}{r^{4}} \frac{\mathrm{d}^{2} G_{n}\left(\alpha_{n} r\right)}{\mathrm{d} r^{2}} G_{n}\left(\alpha_{n} r\right) \mathrm{d} r
$$$$
\frac{d^{2} G_{n}\left(\alpha_{n} r\right)}{\mathrm{d} r^{2}}=\left(\frac{\mathrm{d} z}{\mathrm{~d} r}\right)^{2} \frac{\mathrm{d}^{2} G_{n}(z)}{\mathrm{d} z^{2}}=\alpha_{n}^{2} G_{n}^{\prime \prime}\left(\alpha_{n} r\right)
$$$$
d_{2} g_{n \pm 1}=\int_{r_{1}}^{1} \frac{1}{r^{8}}\left(\alpha_{n \pm 1} r\right)^{2} G_{n \pm 1}^{\prime \prime}\left(\alpha_{n \pm 1} r\right) G_{n}\left(\alpha_{n} r\right) r^{2} \mathrm{~d} r
$$$$
=\int_{r_{1}}^{1} \frac{1}{r^{4}} \frac{\mathrm{d}^{2} G_{n \pm 1}\left(\alpha_{n \pm 1} r\right)}{\mathrm{d} r^{2}} G_{n}\left(\alpha_{n} r\right) \mathrm{d} r
$$$$
d_{2} \mathrm{~g}_{n \pm 2}=\int_{r_{1}}^{1} \frac{1}{r^{8}}\left(\alpha_{n \pm 2} r\right)^{2} G_{n \pm 2}^{\prime \prime}\left(\alpha_{n \pm 2} r\right) G_{n}\left(\alpha_{n} r\right) r^{2} \mathrm{~d} r
$$$$
=\int_{r_{1}}^{1} \frac{1}{r^{4}} \frac{\mathrm{d}^{2} G_{n \pm 2}\left(\alpha_{n \pm 2} r\right)}{\mathrm{d} r^{2}} G_{n}\left(\alpha_{n} r\right) \mathrm{d} r
$$ 
Yukutake and Shimizu Earth, Planets and Space (2018) 70:145

Page 18 of 20

$$
\begin{aligned}
d_{3} g_{n \pm 1} & =\int_{r_{1}}^{1} \frac{1}{r^{8}}\left(\alpha_{n \pm 1} r\right)^{3} G_{n \pm 1}^{\prime \prime \prime}\left(\alpha_{n \pm 1} r\right) G_{n}\left(\alpha_{n} r\right) r^{2} \mathrm{~d} r \\
& =\int_{r_{1}}^{1} \frac{1}{r^{3}} \frac{\mathrm{d}^{3} G_{n \pm 1}\left(\alpha_{n \pm 1} r\right)}{\mathrm{d} r^{3}} G_{n}\left(\alpha_{n} r\right) \mathrm{d} r
\end{aligned}
$$

Appendix: Coefficients in Eqs. (50) and (51)$$
A_{t, n}^{m}=i a_{n-1}^{m}\left(\frac{B_{0}}{\varepsilon^{5} r_{0}}\right) \tilde{A}_{t, n}^{m}, \quad B_{t, n}^{m}=i a_{n-1}^{m}\left(\frac{B_{0}}{\varepsilon^{5} r_{0}}\right) \tilde{B}_{t, n}^{m}
$$$$
\tilde{A}_{t, n}^{m}=\frac{Q_{a n}}{Q_{a d}}, \quad \tilde{B}_{t, n}^{m}=\frac{Q_{b n}}{Q_{b d}}
$$$$
Q_{a d}=(n-1)(n+2) r_{1}^{\prime 2} \frac{1}{\omega-i \omega_{\eta, n+1}} \mathrm{~g} u\left(r_{0}^{\prime}\right)\left(\mathrm{d} r N\left(k r_{1}^{\prime}\right) j_{n}\left(k^{\prime} r_{1}^{\prime}\right)\right.
$$$$
\left.-\mathrm{d} r J\left(k r_{1}^{\prime}\right) n_{n}\left(k^{\prime} r_{1}^{\prime}\right)\right) j_{n}\left(k^{\prime} r_{0}^{\prime}\right)
$$

$$
\begin{aligned}
Q_{a n}= & -\left[( n + 2 ) ( n - 1 ) \frac { 1 } { ( \omega - i \omega _ { \eta , n + 1 } ) ( \omega - i \omega _ { \eta , n - 1 } ) } r _ { 1 } ^ { 2 } \left\{\left(g u\left(r_{1}^{\prime}\right) g v\left(r_{0}^{\prime}\right)\right.\right.\right. \\
& \left.\left.-g u\left(r_{0}^{\prime}\right) g v\left(r_{1}^{\prime}\right)\right) d r J\left(k r_{1}^{\prime}\right)+\left(d g v\left(r_{1}^{\prime}\right) g u\left(r_{0}^{\prime}\right)-d g u\left(r_{1}^{\prime}\right) g v\left(r_{0}^{\prime}\right)\right) j_{n}\left(k^{\prime} r_{1}^{\prime}\right)\right\} n_{n}\left(k^{\prime} r_{0}^{\prime}\right) \\
& -2 i \eta \sigma r_{0}^{\prime} r_{e}^{\prime} 5\left\{n(n+2) t_{v, n}^{m} \frac{1}{\omega-i \omega_{\eta, n+1}} G_{n-1}\left(\alpha_{n-1} r_{1}^{\prime}\right) g u\left(r_{0}^{\prime}\right)\right. \\
& \left.\left.+\left(n^{2}-1\right) t_{u, n}^{m} \frac{1}{\omega-i \omega_{\eta, n-1}} G_{n+1}\left(\alpha_{n+1} r_{1}^{\prime}\right) g v\left(r_{0}^{\prime}\right)\right\} j_{n}\left(k^{\prime} r_{1}^{\prime}\right) n_{n}\left(k^{\prime} r_{0}^{\prime}\right)\right]
\end{aligned}
$$

$$
\begin{aligned}
Q_{b d}= & (n-1)(n+2) r_{1}^{\prime 2} \frac{1}{\omega-i \omega_{\eta, n+1}} \mathrm{~g} u\left(r_{0}^{\prime}\right)\left(\mathrm{d} r N\left(k r_{1}^{\prime}\right) j_{n}\left(k^{\prime} r_{1}^{\prime}\right)\right. \\
& \left.-\mathrm{d} r J\left(k r_{1}^{\prime}\right) n_{n}\left(k r_{1}^{\prime}\right)\right)
\end{aligned}
$$

$$
\begin{aligned}
Q_{b n}= & {\left[( n + 2 ) ( n - 1 ) \frac { 1 } { ( \omega - i \omega _ { \eta , n + 1 } ) ( \omega - i \omega _ { \eta , n - 1 } ) } r _ { 1 } ^ { \prime 2 } \left\{\left(g u\left(r_{1}^{\prime}\right) g v\left(r_{0}^{\prime}\right)\right.\right.\right.} \\
& \left.\left.-g u\left(r_{0}^{\prime}\right) g v\left(r_{1}^{\prime}\right)\right) \mathrm{d} r J\left(k r_{1}^{\prime}\right)+\left(d g v\left(r_{1}^{\prime}\right) g u\left(r_{0}^{\prime}\right)-d g u\left(r_{1}^{\prime}\right) g v\left(r_{0}^{\prime}\right)\right) j_{n}\left(k^{\prime} r_{1}^{\prime}\right)\right\} \\
& -2 i \eta \sigma r_{0}^{\prime} r_{e}^{\prime}\left\{\left\{n(n+2) t_{v, n}^{m} \frac{1}{\omega-i \omega_{\eta, n+1}} G_{n-1}\left(\alpha_{n-1} r_{1}^{\prime}\right) g u\left(r_{0}^{\prime}\right)\right.\right. \\
& \left.+\left(n^{2}-1\right) t_{u, n}^{m} \frac{1}{\omega-i \omega_{\eta, n-1}} G_{n+1}\left(\alpha_{n+1} r_{1}^{\prime}\right) g v\left(r_{0}^{\prime}\right) j_{n}\left(k^{\prime} r_{1}^{\prime}\right)\right]
\end{aligned}
$$




$$
\begin{aligned}
& \tilde{A}_{s, n}^{m}=-\frac{1}{\mathrm{~d} r J J\left(k^{\prime} r_{0}^{\prime}\right)}\left[f G n\left(\alpha_{n} r_{0}^{\prime}\right)+\frac{\mathrm{d} r N N\left(k^{\prime} r_{0}^{\prime}\right)}{\mathrm{d} r N J\left(k^{\prime} r_{1}^{\prime}\right)} f G J\left(r_{1}^{\prime}\right)\right] \\
& \tilde{B}_{s, n}^{m}=\frac{1}{\mathrm{~d} r N J\left(k^{\prime} r_{0}^{\prime}\right)} f G J\left(r_{1}^{\prime}\right) \\
& \mathrm{d} r J J\left(k^{\prime} r^{\prime}\right)=\frac{\mathrm{d}}{\mathrm{d} r^{\prime}}\left[r^{\prime 2} j_{n}\left(k^{\prime} r^{\prime}\right)\right]+n j_{n}\left(k^{\prime} r^{\prime}\right) \\
& \mathrm{d} r N N\left(k^{\prime} r^{\prime}\right)=\frac{\mathrm{d}}{\mathrm{d} r^{\prime}}\left[r^{\prime 2} n_{n}\left(k^{\prime} r^{\prime}\right)\right]+n \cdot n_{n}\left(k^{\prime} r^{\prime}\right) \\
& \mathrm{d} r N J\left(k^{\prime} r^{\prime}\right)=j_{n}\left(k^{\prime} r^{\prime}\right) \frac{\mathrm{d}}{\mathrm{d} r^{\prime}}\left[r^{\prime 2} n_{n}\left(k^{\prime} r^{\prime}\right)\right] \\
& -n_{n}\left(k^{\prime} r^{\prime}\right) \frac{\mathrm{d}}{\mathrm{d} r^{\prime}}\left[r^{\prime 2} j_{n}\left(k^{\prime} r^{\prime}\right)\right] \\
& f G J\left(r^{\prime}\right)=\frac{\mathrm{d}}{\mathrm{d} r^{\prime}}\left[r^{\prime 2} j_{n}\left(k^{\prime} r^{\prime}\right)\right] G_{n}\left(\alpha_{n} r^{\prime}\right) \\
& -j_{n}\left(k^{\prime} r^{\prime}\right) \frac{\mathrm{d}}{\mathrm{d} r^{\prime}}\left[r^{\prime 2} G_{n}\left(\alpha_{n} r^{\prime}\right)\right] \\
& f G n\left(\alpha_{n} r^{\prime}\right)=n G_{n}\left(\alpha_{n} r^{\prime}\right)+\frac{\mathrm{d}}{\mathrm{d} r^{\prime}}\left[r^{\prime 2} G_{n}\left(\alpha_{n} r^{\prime}\right)\right]
\end{aligned}
$$

\section{Appendix: Coefficients in Eqs. (57) and (58)}

$$
\begin{aligned}
\operatorname{gg}_{n}= & \int_{r_{1}}^{1} G_{n}\left(\alpha_{n} r^{\prime}\right) G_{n}\left(\alpha_{n} r^{\prime}\right) r^{\prime 2} \mathrm{~d} r^{\prime} \\
q_{w, n}= & \int_{r_{1}}^{1} \frac{1}{r^{\prime} 4}\left\{U_{n}^{m}+V_{n}^{m}+W_{n}^{m}\right\} G_{n}\left(\alpha_{n} r^{\prime}\right) r^{\prime 2} \mathrm{~d} r^{\prime} \\
U_{n}^{m}= & \frac{1}{2 n-1} \kappa_{n}^{a} t_{u, n-1}^{m} r^{\prime} G_{u, n}^{x}\left(\alpha_{n} r^{\prime}\right) \\
& -\frac{1}{2 n+3} \kappa_{n+1}^{b} t_{v, n+1}^{m} r^{\prime} G_{v, n}^{x}\left(\alpha_{n} r^{\prime}\right) \\
V_{n}^{m}= & \frac{n(n+m+1)}{(2 n+3)(n+1)} \\
& \times\left\{\bar{A}_{t, n+1}^{m} r^{\prime} J_{u, n+1}^{x}\left(k^{\prime} r^{\prime}\right)+\bar{B}_{t, n+1}^{m} r^{\prime} N_{u, n+1}^{x}\left(k^{\prime} r^{\prime}\right)\right\}
\end{aligned}
$$

$$
\begin{aligned}
& W_{g, n}^{m}=4 m s_{n}^{m} \frac{\varepsilon}{r^{\prime}} r^{\prime}\left\{\left(\alpha_{n}^{2} r^{\prime 2}-2\right) G_{n}\left(\alpha_{n} r^{\prime}\right)-2 \alpha_{n} r^{\prime} G_{n}\left(\alpha_{n} r^{\prime}\right)\right\} \\
& W_{g, j}^{m}=4 m s_{n}^{m} \frac{\varepsilon}{r^{\prime}} r^{\prime}\left[\bar{A}_{s, n}^{m} r^{\prime}\left\{\left(k^{\prime 2} r^{\prime 2}-2\right) \bar{j}_{n}\left(k^{\prime} r^{\prime}\right)-2 k^{\prime} r^{\prime} \bar{j}_{n}^{\prime}\left(k^{\prime} r^{\prime}\right)\right\}\right. \\
& \left.+\bar{B}_{s, n}^{m} r^{\prime}\left\{\left(k^{\prime 2} r^{\prime 2}-2\right) \bar{n}_{n}\left(k^{\prime} r^{\prime}\right)-2 k^{\prime} r^{\prime} \bar{n}_{n}^{\prime}\left(k^{\prime} r^{\prime}\right)\right\}\right] \\
& W_{n}^{m}=W_{g, n}^{m}+W_{g, j}^{m} \\
& G_{u, n}^{x}\left(\alpha_{n} r^{\prime}\right)=(n+4) \tilde{G}_{u, n}\left(\alpha_{n} r^{\prime}\right)+2 \alpha_{n} r^{\prime} \tilde{G}_{u, n}^{\prime}\left(\alpha_{n} r^{\prime}\right) \\
& G_{v, n}^{x}\left(\alpha_{n} r^{\prime}\right)=(n-3) \tilde{G}_{v, n}\left(\alpha_{n} r^{\prime}\right)-2 \alpha_{n} r^{\prime} \tilde{G}_{v, n}^{\prime}\left(\alpha_{n} r^{\prime}\right) \\
& \kappa_{n}^{a}=(n-1)(n+1)(n-m) \\
& \kappa_{n}^{b}=(n-1)(n+1)(n+m) \\
& t_{u, n}^{m}=\frac{(n+2)(n+m+1)}{(n+1)(2 n+3)}, \quad t_{v, n}^{m}=\frac{(n-1)(n-m)}{n(2 n-1)} \\
& J_{u, n}^{x}\left(k^{\prime} r^{\prime}\right)=(n-4) \bar{j}_{n}\left(k^{\prime} r^{\prime}\right)-2 k^{\prime} r^{\prime} \bar{j}_{n}^{\prime}\left(k^{\prime} r^{\prime}\right) \\
& N_{u, n}^{x}\left(k^{\prime} r^{\prime}\right)=(n-4) \bar{n}_{n}\left(k^{\prime} r^{\prime}\right)-2 k^{\prime} r^{\prime} \bar{n}_{n}^{\prime}\left(k^{\prime} r^{\prime}\right) \\
& \bar{A}_{s t, n}^{m}=\bar{A}_{s t, n}^{m} j_{n}\left(k^{\prime} r_{0}^{\prime}\right), \quad \tilde{B}_{s t, n}^{m}=\tilde{B}_{s t, n}^{m} j_{n}\left(k^{\prime} r_{0}^{\prime}\right) \\
& \bar{j}_{n}\left(k^{\prime} r^{\prime}\right)=\frac{j_{n}\left(k^{\prime} r^{\prime}\right)}{j_{n}\left(k^{\prime} r_{0}^{\prime}\right)}, \quad \bar{n}_{n}\left(k^{\prime} r^{\prime}\right)=\frac{n_{n}\left(k^{\prime} r^{\prime}\right)}{n_{n}\left(k^{\prime} r_{0}^{\prime}\right)}
\end{aligned}
$$

\section{Appendix: Explicit expression of $S$ in (59) for $m=2$}

$$
\begin{aligned}
S & =B_{0} r^{\prime} r_{0}\left\{\left(\left(5.5 \times 10^{3}-9.4 \times 10^{2} i\right) G_{2}\left(2.10 r^{\prime}\right)\right.\right. \\
& +\left(-1.2 \times 10^{3}+3.8 \times 10^{2} i\right) \bar{j}_{2}\left((2.4-0.58 i) r^{\prime}\right) \\
& +\left(-1.2 \times 10^{2}-9.6 \times 10^{1} i\right) \bar{n}_{2}\left((2.4-0.58 i) r^{\prime}\right) P_{2}^{2}(\cos \theta) e^{i \omega_{2} t} \\
& +\left((9.3-0.74 i) G_{4}\left(4.46 r^{\prime}\right)+(-0.46+1.6 i) \bar{j}_{4}\left((5.0-1.9 i) r^{\prime}\right)\right. \\
& \left.+(-1.2-1.2 i) \bar{n}_{4}\left((5.0-1.9 i) r^{\prime}\right)\right) P_{4}^{2}(\cos \theta) e^{i \omega_{4} t} \\
& +\left((0.29-0.0066 i) G_{6}\left(6.64 r^{\prime}\right)+(0.17+0.082 i) \bar{j}_{6}\left((7.3-3.2 i) r^{\prime}\right)\right. \\
& \left.\left.+(-0.21+0.073 i) \bar{n}_{6}\left((7.3-3.2 i) r^{\prime}\right)\right) P_{6}^{2}(\cos \theta) e^{i \omega_{6} t}+\cdots\right\} e^{2 i \varphi} a_{2}^{2}
\end{aligned}
$$

where

$$
k_{2}^{\prime}=2.6-0.72 i, \quad k_{4}^{\prime}=4.6-1.6 i, \quad k_{6}^{\prime}=6.9-2.6 i .
$$




\section{Publisher's Note}

Springer Nature remains neutral with regard to jurisdictional claims in published maps and institutional affiliations.

Received: 7 May 2018 Accepted: 11 August 2018

Published online: 10 September 2018

\section{References}

Amit H, Choblet G (2012) Mantle-driven geodynamo features-Effects of compositional and narrow D" anomalies. Phys Earth Planet Inter 190:34-43

Amit H, Pais MA (2013) Differences between tangential geostrophy and columnar flow. Geophys J Int 194:145-157

Aubert J, Finlay CC, Founier A (2013) Bottom-up control of geomagnetic secular variation by the Earth's inner core. Nature 502:219-223

Barrois O, Gillet N, Aubert J (2017) Contributions to geomagnetic secular variation from a reanalysis of core surface dynamics. Geophys J Int 211:50-68

Barrois O, Hammer MD, Finlay CC, Martin Y, Gillet N (2018) Assimilation of ground and satellite magnetic measurements: inference of core surface magnetic and velocity field changes. Geophys J Int. https://doi. org/10.1093/gji/ggy297

Bergman MI (1993) Magnetic Rossby waves in a stably stratified layer near the surface of the Earth's outer core. Geophys Astrophys Fluid Dyn 68:151-176

Bloxham J, Jackson A (1991) Fluid flow near the surface of the Earth's outer core. Rev Geophys 29:97-120

Bloxham J, Gubbins D, Jackson A (1989) Geomagnetic secular variation. Phil Trans R Soc Lond A 329:415-502

Braginsky SI (1998) Magnetic Rossby waves in the stratified ocean of the core and topographic core-mantle coupling. Earth Planets Space 50:641-649. https://doi.org/10.1186/BF03352159

Braginsky SI (1999) Dynamics of the stably stratified ocean at the top of the core. Phys Earth Planet Inter 111:21-34

Bretherton FP (1964) Low frequency oscillations trapped near the equator. Tellus 16:181-185

Buffet B (2014) Geomagnetic fluctuations reveal stable stratification at the top of the Earth's core. Nature 507:484-487

Bullard EC, Freedman C, Gellman H, Nixon J (1950) The westward drift of the Earth's magnetic field. Phil Trans R Soc Lond A 234:67-92

Christensen UR, Wicht J (2009) Numerical dynamo simulations in "Core dynamics". In: Schubert G, Olson P (eds) Treatise on geophysics vol 8, 245-282

Finlay CC, Jackson A (2003) Equatorially dominated magnetic field change at the surface of the core. Science 300:2084-2086

Finlay CC, Dumberry M, Chulliat A, Pais MA (2010) Short time scale core dynamics: theory and observation. Space Sci Rev 155:177-218

Finlay CC, Olsen N, Kotslaros S, Gillet N, Tøffner-Clausen L (2016) Recent geomagnetic secular variation from Swarm and ground observatories as estimated in CHAOS-6 geomagnetic field model. Earth Planets Space 68:112. https://doi.org/10.1186/s40623-016-0486-1

Gillet N, Pais MA, Jault D (2009) Ensemble inversion of time-dependent core flow models. Geochem Geophys Geosyst. https://doi.org/10.1029/2008g c002290

Gillet N, Jault D, Finlay CC (2015) Planetary gyre, time-dependent eddies, torsional waves, and equatorial jets at the Earth's core surface. J Geophys Res Solid Earth 120:3991-4013. https://doi.org/10.1002/2014JB011786

Hide R (1966) Free hydromagnetic oscillations of the Earth's core and the theory of the geomagnetic secular variation. Phil Trans R Soc Lond A 259:615-647

Holme R (2015) Large-scale flow in the core "Core dynamics". In: Schubert G, Olson P (eds) Treatise on geophysics, 2nd ed. vol 8, 91-113
Hori K, Teed RJ, Jones CA (2018) The dynamics of magnetic Rossby waves in spherical dynamo simulations: a signature of strong-field dynamos? Phys Earth Planet Inter 276:68-85

Jackson A (2003) Intense equatorial flux spots on the surface of the Earth's core. Nature 424:760-763

Kuang W, Tangborn A, Wei Z, Sabaka T (2009) Constraining a numerical geodynamo model with 100 years of surface observations. Geophys J Int 179:1459-1468

Kuang W, Wei Z, Holme R, Tangborn A (2010) Prediction of geomagnetic field with data assimilation: a candidate secular variation model for IGRF-11. Earth Planets Space 62:775-785. https://doi.org/10.5047/eps.2010.07.008

Longuet-Higgins MS (1968) The eigenfunctions of Laplace's tidal equations over a sphere. Phil Trans R Soc Lond A 262:511-607

Matsuno T (1964) Quasi-geostrophic motions in the equatorial area. J Meteoro Soc Jpn 44:25-43

Pais MA, Jault D (2008) Quasi-geostrophic flows responsible for the secular variation of the Earth's magnetic field. Geophys J Int 173:421-443

Pedlosky J (1987) Geophysical fluid dynamics, 2nd edn. Springer, Berlin, p 710

Roberts PH, Scott S (1965) On the analysis of secular variation, 1, A hydromagnetic constraint: theory. J Geomag Geoelectr 17:137-151

Tangborn A, Kuang W (2015) Geodynamo model and error parameter estimation using geomagnetic data assimilation. Geophys J Int 200:664-675

Yokoyama Y, Yukutake T (1989) Calculation of induced modes of magnetic field in geodynamo problem. J Geomag Geoelectr 41:421-442

Yoshida S, Hamano Y (1993) The westward drift of the geomagnetic field caused by length-of-day variation, and topography of the core-mantle boundary. Geophys J Int 114:696-710

Yukutake T (1981) A stratified core motion inferred from geomagnetic secular variations. Phys Earth Planet Inter 24:253-258

Yukutake T, Shimizu H (2015) Drifting and standing field in the geomagnetic field for the past 400 years. Phys Earth Planet Inter 248:63-72

Yukutake T, Shimizu H (2016) On the latitude dependence of drift velocity of the geomagnetic field. Phys Earth Planet Inter 257:28-39

Yukutake T, Tachinaka H (1968a) Non-dipole part of the earth's magnetic field. Bull Earthq Res Inst Univ Tokyo 46:1027-1074

Yukutake T, Tachinaka H (1968b) The westward drift of the geomagnetic secular variation. Bull Earthq Res Inst Univ Tokyo 46:1075-1102

Yukutake T, Tachinaka H (1969) Separation of the Earth's magnetic field into the drifting and the standing parts. Bull Earthq Res Inst Univ Tokyo 47:65-97

Yukutake T, Yokoyama Y (1988) Types of liquid core motions compatible with the observed geomagnetic secular variation of several hundred years. In: Smylie DE, Hide R (eds) Structure and dynamics of earth's deep interior. Geophys Monograph (AGU), vol 46, pp 95-106

Zhang K (1993) On equatorially trapped boundary inertial waves. J Fluid Mech 248:203-217

\section{Submit your manuscript to a SpringerOpen ${ }^{\circ}$ journal and benefit from:}

- Convenient online submission

- Rigorous peer review

- Open access: articles freely available online

- High visibility within the field

- Retaining the copyright to your article

Submit your next manuscript at $\boldsymbol{\nabla}$ springeropen.com 